\title{
The Effects of Pharmacological and Non-Pharmacological Interventions on Symptom Management and Quality of Life among Breast Cancer Survivors Undergoing Adjuvant Endocrine Therapy: A Systematic Review
}

\author{
Carmen W.H. Chan ${ }^{1}$, , Daria Tai ${ }^{2}$, Stephanie Kwong ${ }^{3}$, Ka Ming Chow ${ }^{1}$, Dorothy N.S. Chan ${ }^{1}$ \\ and Bernard M.H. Law ${ }^{1, *}$ \\ 1 The Nethersole School of Nursing, Faculty of Medicine, The Chinese University of Hong Kong, Hong Kong, \\ China; whchan@cuhk.edu.hk (C.W.H.C.); kmchow@cuhk.edu.hk (K.M.C.); dorothycns@cuhk.edu.hk (D.N.S.C.) \\ 2 Department of Psychology, The University of British Columbia, Vancouver, BC V6T 1Z4, Canada; \\ daria.tai@alumni.ubc.ca \\ 3 Department of Pharmacology and Toxicology, University of Toronto, Toronto, ON M5S 1A8, Canada; \\ swykwong200@gmail.com \\ * Correspondence: bernardlaw@cuhk.edu.hk; Tel.: +85-239-435-522
}

Received: 19 March 2020; Accepted: 18 April 2020; Published: 24 April 2020

\begin{abstract}
Breast cancer survivors need to undergo adjuvant endocrine therapy after completion of curative treatments to prevent disease recurrence. These individuals often experience symptoms which are detrimental to their quality of life (QOL). Implementation of interventions for effective symptom management among these survivors is warranted. This review provides an overview of studies on the effectiveness of the previously developed interventions for breast cancer survivors undergoing adjuvant endocrine therapy on symptom alleviation and enhancement of QOL or health-related QOL (HRQOL). Five electronic databases were employed in the literature search. Study selection, data extraction and critical appraisal of the included studies were conducted by three authors independently. Twenty-four studies were included. Both pharmacological and non-pharmacological interventions are effective in addressing the symptoms associated with adjuvant endocrine therapy among the breast cancer survivors, and in improving their QOL, although discrepancies were noted between the studies in terms of the significance of these effects. Pharmacological and non-pharmacological interventions can be effective for symptom management among breast cancer survivors. Their implementation is recommended for effective survivorship care for these individuals. Further research on intervention development for breast cancer survivors is recommended to provide further evidence for the utility of the explored interventions in survivorship care for these patients.
\end{abstract}

Keywords: Breast cancer; endocrine therapy; hormonal therapy; intervention; quality of life; symptom; survivorship care

\section{Introduction}

Breast cancer patients, who have completed primary treatments such as surgery, chemotherapy and radiotherapy, require additional therapies for preventing recurrence of the disease. Adjuvant endocrine therapies, which encompass hormonal or endocrine therapy using drugs such as tamoxifen and aromatase inhibitors (AI), are known to serve this purpose [1-5]. These drugs are prescribed to breast cancer patients after the completion of primary treatment, which need to be taken daily for a duration of five years for the therapy to be effective [6]. Premature discontinuation of adjuvant 
endocrine therapy was reported to be prevalent among cancer survivors $[1,7]$. This highlights that patient non-adherence to adjuvant therapies is a major issue.

Previous studies demonstrated that patient non-adherence to adjuvant endocrine therapy is primarily caused by the adverse side effects on patients' quality of life (QOL) that are associated with such therapy $[8,9]$. These common side effects may include hot flashes, night sweats, fatigue, sleep disturbance, sexual dysfunction, joint stiffness, joint dysfunction and joint pain [9-13]. These symptoms may also negatively affect patients' functional ability. For example, muscle pain caused by aromatase inhibitor-based therapies could lead to physical impairment [14]. Moreover, adverse effects associated with adjuvant endocrine therapy were demonstrated to lead to patients' early discontinuation of such adjuvant treatment [15].

Non-adherence to and/or early discontinuation of adjuvant endocrine therapy would exhibit negative effects on the well-being of breast cancer survivors. It could lead to an increase in breast cancer mortality and an increased risk of cancer recurrence [1,16]. This is compounded with higher costs incurred for cancer treatment and poorer QOL $[16,17]$. The development of effective strategies for the management of side effects associated with adjuvant endocrine therapy is therefore of paramount importance. Over the past years, there has been an increasing number of published studies reporting the development of interventions which would be effective in addressing the aforementioned adjuvant endocrine therapy-induced symptoms. Given the potential of these interventions in improving the well-being of breast cancer survivors, conduction of a systematic review that examines the findings of these interventional studies and summarizes the major components of these interventions is worthwhile.

Previously, several reviews have been published, focusing on the various types of interventions that target specific symptoms associated with adjuvant endocrine therapy [18-22]. To the best of our knowledge, a systematic review that examines the effectiveness of the different types of interventions on various known adjuvant endocrine therapy-induced symptoms, overall QOL and functional ability of patients is yet to be conducted. The aim of the present review is to provide an overview on the findings of randomized control trials reporting the effectiveness of various types of interventions targeted for breast cancer survivors undergoing adjuvant endocrine therapy on the improvement of their QOL or health-related QOL (HRQOL) and functional ability. The review also examines the effect of these interventions on alleviating common symptoms reported to be caused by adjuvant endocrine therapy, including hot flashes, night sweats, fatigue, sleep disturbance, sexual dysfunction, joint stiffness, joint dysfunction and joint pain. Findings of this review may provide strategic directions to intervention development for breast cancer patients who are in high need for effective management of the aforementioned undesirable side effects of adjuvant endocrine therapy and reduce their non-adherence to the therapy.

\section{Methods}

\subsection{Search Strategy}

We performed a comprehensive literature search in November 2019 to identify articles reporting interventions or programs developed for breast cancer survivors undergoing adjuvant endocrine therapy, from date of inception to November 2019. This enabled a comprehensive review of the articles that report the effectiveness of the relevant interventions published previously. Five databases, including PubMed, OVID MEDLINE (since 1946), EMBASE (since 1910), PsycINFO (since 1806), and CINAHL Complete, were used for the search. We employed the search strategy that is set out in Table 1. 
Table 1. The Search Strategy.

\begin{tabular}{|c|}
\hline "Breast cancer" OR “carcinoma" OR “neoplasm" OR “malignancy" \\
\hline AND \\
\hline "survivorship" OR “survivor" OR “survivors" OR "patient" OR “patients" \\
\hline AND \\
\hline $\begin{array}{l}\text { "Tamoxifen" OR “Aromatase inhibitor" OR "endocrine therapy" OR "hormone therapy" OR "hormonal } \\
\text { therapy" }\end{array}$ \\
\hline AND \\
\hline $\begin{array}{l}\text { "Intervention" OR “therapy" OR “program" OR "programme" OR "pharmacological" OR } \\
\text { "non-pharmacological" }\end{array}$ \\
\hline AND \\
\hline 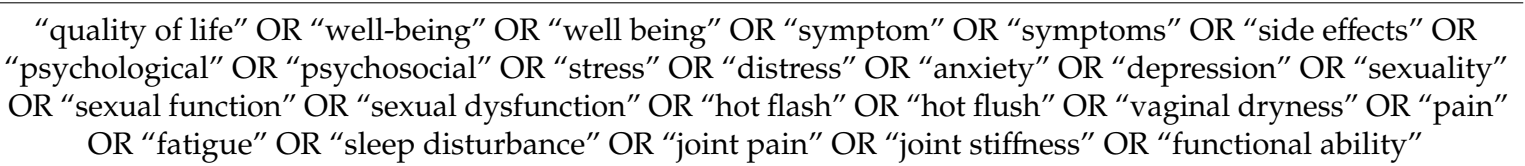 \\
\hline
\end{tabular}

\subsection{Inclusion and Exclusion Criteria}

To be included in the review, research articles should describe interventions developed for breast cancer survivors who are: (1) not undergoing curative treatments such as chemotherapy and radiotherapy; and (2) on adjuvant endocrine therapy. Included studies should report interventions that aim to improve one or more of the following outcomes: (1) endocrine symptoms (vaginal dryness, night sweats and hot flashes); (2) sexual symptoms; (3) joint symptoms (joint pain and stiffness); (4) fatigue; (5) sleep problems; and (6) QOL or HRQOL. Only studies with a randomized control trial (RCT) design and those written in English are included. Studies that included samples not entirely comprising participants undergoing adjuvant endocrine therapy at the time of receiving the reported intervention, yet without the use of stratified analysis, were excluded.

\subsection{Data Extraction and Summary}

Using the above search strategy and inclusion and exclusion criteria, two authors independently screened the titles and abstracts of the extracted studies, and selected the articles for inclusion in the review. The resultant list of included articles was confirmed by a third author. The full-text of the included articles was further examined by the three authors to confirm the eligibility of the articles to be included in the review.

Upon finalization of the list of included articles, two authors independently performed data extraction, which was subjected to verification by one author to ensure accuracy. These data included settings of the reported interventions, characteristics of the participants, content of intervention, assessed outcomes of interest, data collection time points, instruments used for outcome assessments, and study findings. Disagreements between the authors on the extracted data were resolved through discussion until a consensus was made.

In this review, the significance of intervention effects on the outcomes of interest was indicated with $p$ values.

\subsection{Critical Appraisal}

The assessment of the methodological quality of the selected studies was performed using "The Quality Assessment Tool for Quantitative Studies" in the Effective Public Health Practice Project (EPHPP). The methodology on how the assessment tool is used for critical appraisal of the included studies was previously described [23]. Briefly, a rating of either strong, moderate or weak was given for each study on six categories involved in the assessment, namely selection bias, study design, 
confounders, blinding, data collection methods and withdrawals and dropouts. The elements assessed in each of these categories were described previously [23]. Such ratings were performed based on the assessment criteria set out by the EPHPP [24]. Based on the rating for each category, a global rating was given. A strong global rating indicates the study received no weak ratings in any category and has four or more strong-rated categories. A study was assigned a global rating of "moderate" if it possessed one weak category and/or fewer than four strong-rated categories. A study with more than one category having a weak rating was given a "weak" global rating. This critical appraisal was performed by three authors independently. Disagreements in rating assignments were resolved through discussion between the three authors.

\section{Results}

\subsection{Search Results}

The PRISMA diagram is presented as Figure 1. Briefly, the search strategy (Table 1) enabled the identification of 8994 articles. After the removal of 2033 articles retrieved in duplications in multiple databases, we screened the abstracts of the remaining 6961 articles. We excluded 6268 articles not reporting an RCT, 165 articles that were not original articles and 272 articles not published in English. A total of 232 articles were further excluded as they did not report an intervention or they did not involve the participant type and/or outcome measures indicated in the inclusion criteria. The search resulted in the inclusion of 24 articles in the present review.

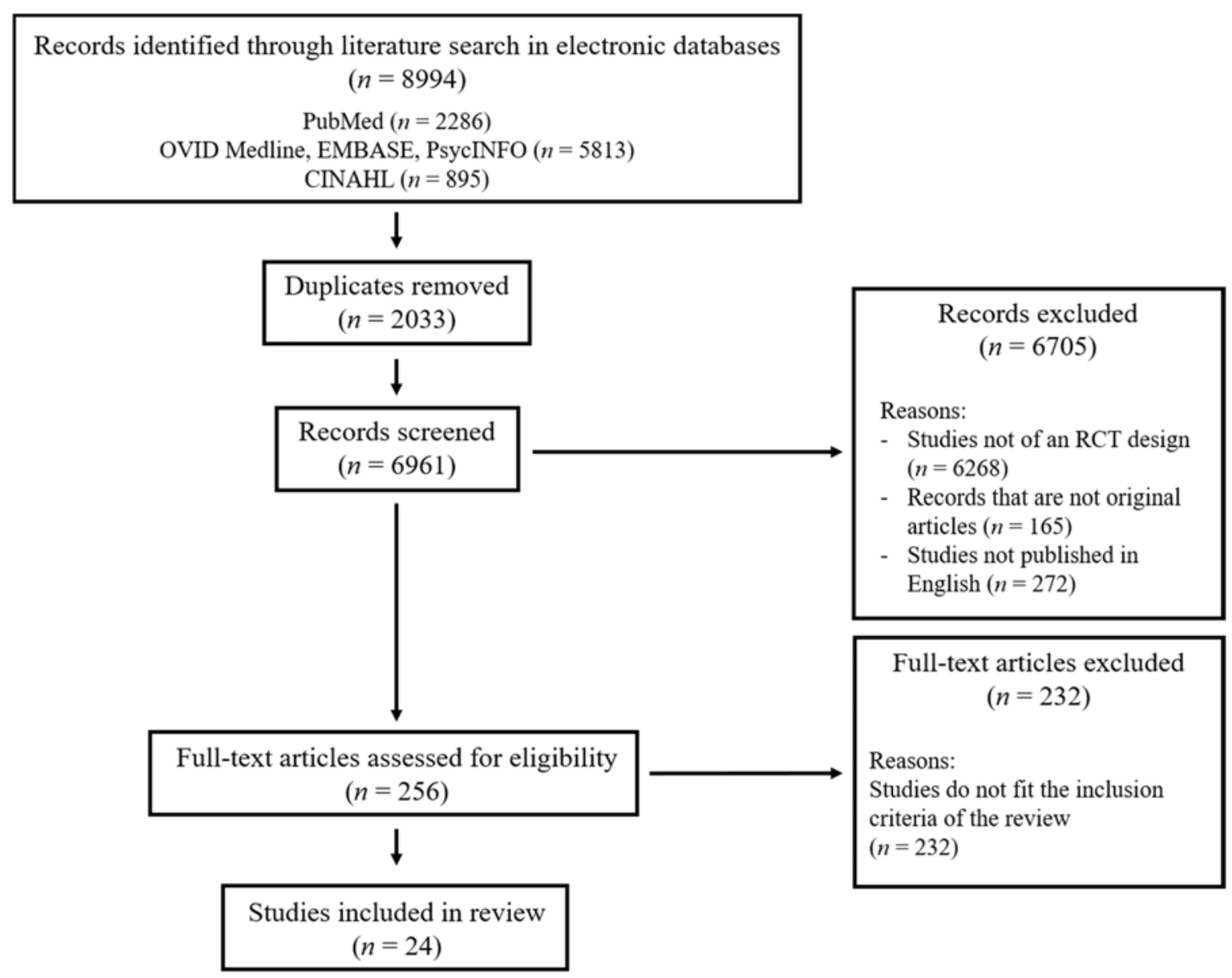

Figure 1. The PRISMA Diagram. 


\subsection{Methodological Quality of Included Studies}

Fifty-four percent $(n=13)$ of included studies were given an overall rating as weak, $25 \%(n=6)$ as moderate, and $21 \%(n=5)$ as strong. Around $67 \%$ of the included studies $(n=16)$ were rated moderate for selection bias. These studies utilized an appropriate sampling method to generate a representative sample of the population. A number of studies did not report the number of participants approached during subject recruitment, making it impossible to determine the percentage of participants who provided their consent to study participation.

Considerable proportion of the included studies were rated as strong in the study design $(n=14$, $58 \%$ ) where most studies utilized an appropriate randomization technique. Eighty-eight percent of studies $(n=21)$ were rated as strong in the confounders category, where they either demonstrated no significant between-group differences in baseline characteristics or confounders were addressed in the case where such differences were observed. Additionally, eight studies (33\%) were rated as strong in the blinding category, where they involved participants and assessors who were blinded to the treatment allocation. Most of the studies reported a strong data collection method ( $n=22,92 \%)$, with the data collection tools used in these studies demonstrated to be reliable and valid. Fifty-eight percent $(n=14)$ of studies were rated as strong in the withdrawals and dropouts category, where they reported that at least $80 \%$ of the participants had completed the study. Table 2 shows a tabular summary of the methodological quality ratings of each included study. In summary, $46 \%$ of the included studies were either rated strong or moderate in the appraisal. Weaknesses of the methodological quality among these studies were primarily contributed by: (1) the impossibility of blinding the participants and/or outcome assessors owing to the nature of the intervention; and (2) the lack of reporting on the number of participants approached during subject recruitment and the method of randomization conducted.

Table 2. The methodological quality of the included studies.

\begin{tabular}{cccccccc}
\hline $\begin{array}{c}\text { Author/Year } \\
\text { [25-48] }\end{array}$ & $\begin{array}{c}\text { Selection } \\
\text { Bias }\end{array}$ & $\begin{array}{c}\text { Study } \\
\text { Design }\end{array}$ & Confounders & Blinding & $\begin{array}{c}\text { Data Collection } \\
\text { Method }\end{array}$ & $\begin{array}{c}\text { Withdrawals } \\
\text { and Dropouts }\end{array}$ & Overall \\
\hline $\begin{array}{c}\text { Advani et al., } \\
2017 \text { [33] }\end{array}$ & Moderate & Strong & Strong & Weak & Strong & Strong & Moderate \\
\hline $\begin{array}{c}\text { Baker et al., } \\
\text { 2018 [43] }\end{array}$ & Moderate & Strong & Strong & Moderate & Strong & Strong & Strong \\
\hline $\begin{array}{c}\text { Conejo et al., } \\
2018 \text { [47] }\end{array}$ & Moderate & Strong & Strong & Weak & Strong & Strong & Moderate \\
\hline $\begin{array}{c}\text { Crew et al., } \\
2007 \text { [42] }\end{array}$ & Moderate & Weak & Weak & Weak & Strong & Strong & Weak \\
\hline $\begin{array}{c}\text { de Sousa et al., } \\
2019 \text { [25] }\end{array}$ & Moderate & Weak & Strong & Strong & Strong & Moderate & Moderate \\
\hline $\begin{array}{c}\text { Goldberg et al., } \\
\text { 1994 [29] }\end{array}$ & Weak & Weak & Strong & Strong & Weak & Weak & Weak \\
\hline $\begin{array}{c}\text { Henry et al., } \\
2018 ~[34]\end{array}$ & Weak & Weak & Strong & Strong & Strong & Strong & Weak \\
\hline $\begin{array}{c}\text { Hershman et al., } \\
2015 \text { [39] }\end{array}$ & Weak & Weak & Strong & Strong & Strong & Moderate & Weak \\
\hline $\begin{array}{c}\text { Hershman et al., } \\
2018 ~[40]\end{array}$ & Moderate & Weak & Weak & Weak & Strong & Strong & Weak \\
\hline $\begin{array}{c}\text { Heudel et al., } \\
2019 \text { [26] }\end{array}$ & Moderate & Strong & Strong & Strong & Strong & Strong & Strong \\
\hline $\begin{array}{c}\text { Irwin et al., } \\
2015[38]\end{array}$ & Moderate & Strong & Strong & Weak & Strong & Strong & Weak \\
\hline
\end{tabular}


Table 2. Cont.

\begin{tabular}{|c|c|c|c|c|c|c|c|}
\hline \multirow[b]{2}{*}{$\begin{array}{c}\text { Author/Year } \\
\text { [25-48] }\end{array}$} & \multicolumn{7}{|c|}{ Methodological Quality Rating (EPHPP) } \\
\hline & $\begin{array}{c}\text { Selection } \\
\text { Bias }\end{array}$ & $\begin{array}{c}\text { Study } \\
\text { Design }\end{array}$ & Confounders & Blinding & $\begin{array}{l}\text { Data Collection } \\
\text { Method }\end{array}$ & $\begin{array}{l}\text { Withdrawals } \\
\text { and Dropouts }\end{array}$ & Overall \\
\hline $\begin{array}{l}\text { Keshavarzi et al., } \\
2019[31]\end{array}$ & Moderate & Strong & Strong & Strong & Strong & Strong & Strong \\
\hline $\begin{array}{l}\text { Kimmick et al., } \\
2006 \text { [27] }\end{array}$ & Weak & Weak & Strong & Strong & Strong & Moderate & Weak \\
\hline $\begin{array}{l}\text { Mann et al., } \\
2012 \text { [30] }\end{array}$ & Moderate & Strong & Strong & Moderate & Strong & Strong & Strong \\
\hline $\begin{array}{l}\text { Mao et al., } \\
2014 \text { [46] }\end{array}$ & Weak & Strong & Strong & Weak & Strong & Strong & Weak \\
\hline $\begin{array}{l}\text { Nyrop et al., } \\
2017 \text { [35] }\end{array}$ & Moderate & Weak & Weak & Weak & Strong & Moderate & Weak \\
\hline $\begin{array}{c}\text { Oh et al., } 2013 \\
{[41]}\end{array}$ & Moderate & Strong & Strong & Weak & Strong & Strong & Moderate \\
\hline $\begin{array}{l}\text { Pandya et al., } \\
2000 \text { [28] }\end{array}$ & Moderate & Strong & Strong & Strong & Strong & Moderate & Strong \\
\hline $\begin{array}{l}\text { Paulo et al., } \\
2019 \text { [32] }\end{array}$ & Moderate & Weak & Strong & Weak & Strong & Moderate & Weak \\
\hline $\begin{array}{c}\text { Peppone et al., } \\
2015 \text { [45] }\end{array}$ & Weak & Strong & Strong & Weak & Weak & Weak & Weak \\
\hline $\begin{array}{l}\text { Rogers et al., } \\
2009 \text { [36] }\end{array}$ & Moderate & Strong & Strong & Weak & Strong & Strong & Moderate \\
\hline $\begin{array}{l}\text { Rogers et al., } \\
2009 \text { [37] }\end{array}$ & Moderate & Strong & Strong & Weak & Strong & Strong & Moderate \\
\hline $\begin{array}{c}\text { Rogers et al., } \\
2017 \text { [48] }\end{array}$ & Weak & Strong & Strong & Moderate & Strong & Weak & Weak \\
\hline $\begin{array}{l}\text { Zhao et al., } \\
2012 \text { [44] }\end{array}$ & Weak & Weak & Strong & Weak & Strong & Weak & Weak \\
\hline
\end{tabular}

\subsection{Effects of Interventions on Patient Outcomes}

The characteristics of the included studies, including the content of the interventions reported, are summarized in reverse chronological order in Table 3. Our review identified pharmacological interventions, four major types of non-pharmacological interventions (physical activity interventions, psychotherapeutic interventions, dietary interventions and acupuncture interventions) and other miscellaneous types of interventions that have previously been developed to aid management of adjuvant endocrine therapy-induced symptoms among breast cancer survivors. This section of the review summarizes the findings of the studies reporting their effect on the alleviating the various groups of adjuvant endocrine therapy-induced symptoms as menopausal symptoms, impaired sexual function, joint symptoms, fatigue and sleep disturbance, as well as the enhancement of survivors' QOL, HRQOL and functional status. 
Table 3. Characteristics of the included studies.

\begin{tabular}{|c|c|c|c|c|c|c|}
\hline $\begin{array}{l}\text { Author/Year/ } \\
\text { Country }\end{array}$ & Settings & Participants/Sample Size & $\begin{array}{c}\text { Intervention } \\
\text { Type }\end{array}$ & Intervention & $\begin{array}{c}\text { Assessed Outcomes of } \\
\text { Interest/Data Collection Time } \\
\text { Points }\end{array}$ & $\begin{array}{l}\text { Instruments for Outcome } \\
\text { Assessments }\end{array}$ \\
\hline $\begin{array}{l}\text { de Sousa } \\
\text { Vieira et al., } \\
2019 \text { [25]; Brazil }\end{array}$ & Not specified & $\begin{array}{l}\text { Breast cancer patients (stage } \\
\text { unknown) undergoing adjuvant } \\
\text { hormone therapy using either } \\
\text { tamoxifen or anastrozole. } \\
\begin{array}{l}N=40 \\
\text { Intervention: } 20 \\
\text { Control: } 20 \\
\text { Duration of adjuvant endocrine } \\
\text { therapy undergone by subjects: } \\
\text { All participants: At least } 3 \text { months } \\
\text { (not specified for each group) }\end{array}\end{array}$ & $\begin{array}{l}\text { Pharmacological } \\
\text { intervention }\end{array}$ & $\begin{array}{l}\text { Pharmacological intervention with } \\
\text { tablets of a medicinal plant extract } \\
\text { (Paullinia cupana or Guarana) } \\
\text { Intervention: } \\
\text { Intake of tablets containing } 37.5 \text { mg of } \\
\text { an active ingredient of guarana (PC-18) } \\
\text { twice daily for } 4 \text { weeks. } \\
\text { Control: } \\
\text { Intake of placebo tablets for } 4 \text { weeks }\end{array}$ & 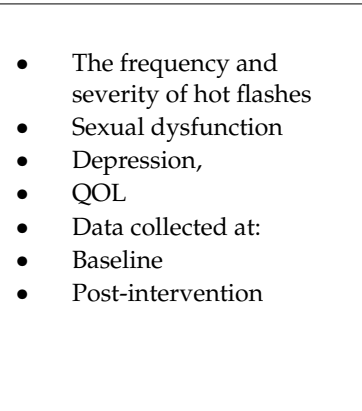 & $\begin{array}{ll}\text { - } & \text { Daily hot flashes diary } \\
\text { - } & \text { Srizona Sex Experience } \\
\text { - } & \text { The Beck } \\
\text { - } & \text { Depression Scale } \\
\text { European Organization } \\
\text { for Research and } \\
\text { Treatment of Cancer Core } \\
\text { Quality of Life } \\
\text { Questionnaire C30 }\end{array}$ \\
\hline $\begin{array}{l}\text { Heudel et al., } \\
2019 \text { [26]; France }\end{array}$ & Not specified & $\begin{array}{l}\text { Patients with localized breast cancer } \\
\text { (stage unknown), receiving adjuvant } \\
\text { endocrine therapy with tamoxifen or } \\
\text { aromatase inhibitors } \\
N=138 \\
\text { Intervention: } 65 \\
\text { Control: } 73 \\
\text { Duration of adjuvant endocrine } \\
\text { therapy undergone by subjects: } \\
\text { All participants: At least } 1 \text { month } \\
\text { (not specified for each group) }\end{array}$ & $\begin{array}{l}\text { Pharmacological } \\
\text { intervention }\end{array}$ & 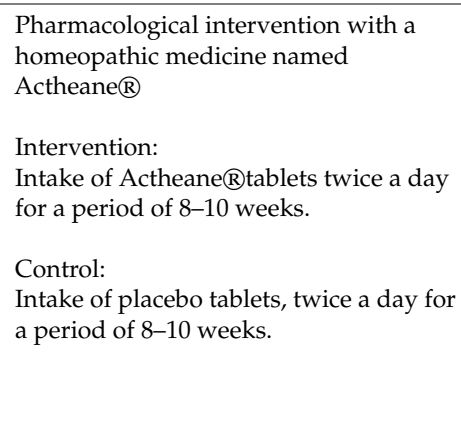 & $\begin{array}{l}\text { - Hot flashes score } \\
\text { - } \quad \text { Frequency of hot flashes } \\
\text { on QOLL } \\
\text { Data collected at: } \\
\text { - } \quad \text { Baseline } \\
\text { - } \quad 4 \text { weeks after } \\
\text { randomization (T1) } \\
\text { - } 8 \text { weeks after } \\
\text { randomization (T2) }\end{array}$ & $\begin{array}{ll}\text { - } & \text { Hot flush self-report diary } \\
\text { Hot Flash Related Daily } \\
\text { Interference Scale }\end{array}$ \\
\hline
\end{tabular}


Table 3. Cont

\begin{tabular}{|c|c|c|c|c|c|c|}
\hline $\begin{array}{l}\text { Author/Year/ } \\
\text { Country }\end{array}$ & Settings & Participants/Sample Size & $\begin{array}{l}\text { Intervention } \\
\text { Type }\end{array}$ & Intervention & $\begin{array}{c}\text { Assessed Outcomes of } \\
\text { Interest/Data Collection Time } \\
\text { Points } \\
\end{array}$ & $\begin{array}{l}\text { Instruments for Outcome } \\
\text { Assessments }\end{array}$ \\
\hline $\begin{array}{l}\text { Keshavarzi et al., } \\
2019 \text { [31]; Iran }\end{array}$ & $\begin{array}{l}\text { Local breast } \\
\text { clinic }\end{array}$ & $\begin{array}{l}\text { Patients with stage I-II breast cancer, } \\
\text { undergoing tamoxifen therapy } \\
N=96 \\
\text { Vitamin D group: } 32 \\
\text { Vitamin E group: } 32 \\
\text { Placebo group: } 32 \\
\text { Duration of adjuvant endocrine } \\
\text { therapy undergone by subjects: } \\
\text { Vitamin D group: } 23.6 \pm 17.5 \text { months } \\
\text { Vitamin E group: } 30.4 \pm 21 \text { months } \\
\text { Control group: } 16.6 \pm 12.5 \text { months }\end{array}$ & $\begin{array}{l}\text { Miscellaneous } \\
\text { intervention }\end{array}$ & $\begin{array}{l}\text { Vitamin } \mathrm{D} / \text { vitamin } \mathrm{E} \text { vaginal } \\
\text { suppository intervention } \\
\text { Intervention: } \\
\text { Participants were provided with vaginal } \\
\text { suppositories supplemented with either } \\
0.025 \mathrm{mg} \text { vitamin } \mathrm{D} \text { or } 1 \mathrm{mg} \text { vitamin } \mathrm{E} \text {, } \\
\text { and were instructed to insert one } \\
\text { suppository into the vagina every day } \\
\text { before bedtime, over the course of } 8 \\
\text { weeks. Telephone reminders were given } \\
\text { every } 3 \text { days. } \\
\text { Control: } \\
\text { The use of vaginal suppositories } \\
\text { supplemented with placebo, instead of } \\
\text { vitamin } \mathrm{D} / \mathrm{E} \text { vaginal suppositories. }\end{array}$ & $\begin{array}{l}\text { - Level of vaginal atrophy } \\
\text { Data collected at: } \\
\text { - } \quad \text { Baseline } \\
\text { - } 2 \text { weeks after start } \\
\text { of intervention } \\
\text { - } 4 \text { weeks after start } \\
\text { of intervention } \\
\text { - } 8 \text { weeks after start } \\
\text { of intervention }\end{array}$ & $\begin{array}{l}\text { - The genitourinary } \\
\text { atrophy } \\
\text { self-assessment tool }\end{array}$ \\
\hline $\begin{array}{l}\text { Paulo et al., } 2019 \\
\text { [32]; Brazil }\end{array}$ & Not specified & $\begin{array}{l}\text { Breast cancer survivors previously } \\
\text { diagnosed with stage I-IIIA cancer, } \\
\text { undergoing aromatase inhibitor } \\
\text { therapy } \\
N=36 \\
\text { Intervention: } 18 \\
\text { Control: } 18 \\
\text { Duration of adjuvant endocrine } \\
\text { therapy undergone by subjects: } \\
\text { Intervention group: } 19.3 \pm 8.3 \\
\text { months } \\
\text { Control group: } 17.9 \pm 11.2 \text { months }\end{array}$ & $\begin{array}{l}\text { Physical activity } \\
\text { intervention }\end{array}$ & $\begin{array}{l}\text { Supervised combined exercise training } \\
\text { intervention } \\
\text { Intervention: } \\
\text { - Participation in a 9-month exercise } \\
\text { program, with sessions held } 3 \\
\text { times a week, containing resistance } \\
\text { training exercises, aerobic exercises } \\
\text { and stretching exercises } \\
\text { Attendance to 90-min health } \\
\text { education lectures once a month, } \\
\text { with topics on breast cancer, health } \\
\text { promotion, quality of life, physical } \\
\text { activity, well being and } \\
\text { mental health } \\
\text { Control: } \\
\text { Participation of } 45-\text { min sessions of } \\
\text { stretching and relaxation exercises twice } \\
\text { a week, over the course of } 9 \text { months. }\end{array}$ & $\begin{array}{l}\text { - } \quad \text { QOL } \\
\text { - } \quad \text { Pain } \\
\text { - } \quad \text { Sleep disturbance } \\
\text { - } \text { Sexual functioning } \\
\text { Data collected at: } \\
\text { - } \quad \text { Baseline } \\
\text { - } 12 \text { weeks after start of } \\
\text { - } \quad 24 \text { weeks after start of } \\
\text { - } \quad 36 \text { weeks after start of } \\
\text { intervention (T3) }\end{array}$ & $\begin{array}{ll}\text { - } & \text { European Organization } \\
\text { for Research and } \\
\text { Treatment of Cancer Core } \\
\text { Quality of Life } \\
\text { Questionnaire C30 } \\
\text { (EORTC-CLC-C30) } \\
\text { - European Organization } \\
\text { for Research and } \\
\text { Treatment of breast } \\
\text { cancer module } \\
\text { - Short Form 36 Health } \\
\text { Survey (SF-36) }\end{array}$ \\
\hline
\end{tabular}


Table 3. Cont

\begin{tabular}{|c|c|c|c|c|c|c|}
\hline $\begin{array}{c}\text { Author/Year/ } \\
\text { Country }\end{array}$ & Settings & Participants/Sample Size & $\begin{array}{l}\text { Intervention } \\
\text { Type }\end{array}$ & Intervention & $\begin{array}{c}\text { Assessed Outcomes of } \\
\text { Interest/Data Collection Time } \\
\text { Points }\end{array}$ & $\begin{array}{l}\text { Instruments for Outcome } \\
\text { Assessments }\end{array}$ \\
\hline $\begin{array}{l}\text { Baker et al., } 2018 \\
\text { [43]; Australia }\end{array}$ & $\begin{array}{l}\text { Local } \\
\text { institution or } \\
\text { hospital } \\
\text { department }\end{array}$ & $\begin{array}{l}\text { Breast cancer patients (stage } \\
\text { unknown) undergoing aromatase } \\
\text { inhibitor therapy } \\
N=31 \\
\text { Intervention: } 14 \\
\text { Control: } 17 \\
\text { Duration of adjuvant endocrine } \\
\text { therapy undergone by subjects: } \\
\text { Intervention group: } 19 \text { months } \\
\text { (median) } \\
\text { Control group: } 3 \text { months (median) }\end{array}$ & $\begin{array}{l}\text { Miscellaneous } \\
\text { intervention }\end{array}$ & $\begin{array}{l}\text { Whole body vibration intervention } \\
\text { Intervention: } \\
\text { - Attendance to sessions involving } \\
\text { exposure to low-frequency and } \\
\text { low-magnitude whole body } \\
\text { vibration via a vibration platform. } \\
\text { Each session lasted for } 20 \mathrm{~min} \text {, and } \\
\text { was held } 3 \text { times a week for a } \\
\text { duration of } 12 \text { weeks. } \\
\text { Control: } \\
\text { Usual care }\end{array}$ & $\begin{array}{l}\text { - Functional ability } \\
\text { - } \quad \text { Joint symptoms } \\
\text { Data collected at: } \\
\text { - } \quad \begin{array}{l}\text { Baseline } \\
\text { - Immediate post- } \\
\text { intervention }\end{array}\end{array}$ & $\begin{array}{ll}\text { - } & \text { Chair rise and stair climb } \\
\text { (objective measure of } \\
\text { functional ability) } \\
\text { - } \quad \text { Functional Assessment of } \\
\text { Cancer Therapy-Fatigue } \\
\text { - Western Ontario and } \\
\text { McMaster Universities } \\
\text { Arthritis Index }\end{array}$ \\
\hline $\begin{array}{l}\text { Conejo et al., } \\
2018 \text { [47]; Spain }\end{array}$ & Not specified & $\begin{array}{l}\text { Breast cancer survivors previously } \\
\text { diagnosed with stage I-IIIA cancer, } \\
\text { undergoing aromatase inhibitor } \\
\text { therapy } \\
N=40 \\
\text { Intervention: } 20 \\
\text { Control: } 20 \\
\text { Duration of adjuvant endocrine } \\
\text { therapy undergone by subjects: } \\
\text { Intervention group: } 15.25 \text { months } \\
\text { (mean) } \\
\text { Control group: } 19.55 \text { months (mean) }\end{array}$ & $\begin{array}{l}\text { Miscellaneous } \\
\text { intervention }\end{array}$ & $\begin{array}{l}\text { Neuromuscular taping intervention } \\
\text { Intervention: } \\
\text { - Application of strips of } \\
\text { neuromuscular taping over the } \\
\text { body parts where pain was felt for } \\
7 \text { days } \\
\text { - Provision of health advice, } \\
\text { focusing on active lifestyles } \\
\text { Control: } \\
\text { Sham neuromuscular taping over body } \\
\text { parts where pain was felt for } 7 \text { days } \\
\text { Provision of health advice, focusing on } \\
\text { active lifestyles }\end{array}$ & $\begin{array}{ll}\text { - } & \text { Mood state } \\
\text { - } & \text { QOL } \\
\text { - } & \text { Pain } \\
\text { - } & \text { Sleep disturbance } \\
& \\
\text { Data collected at: } \\
\text { - } \quad \text { Baseline } \\
\text { - } \quad 1 \text { week after start of } \\
\text { - } \quad 5 \text { weentention (T1) } \\
\quad \text { intervention (T2) }\end{array}$ & $\begin{array}{ll}\text { - } & \text { Profile of Mood States } \\
\text { European Organization } \\
\text { for Research and } \\
\text { Treatment of Cancer Core } \\
\text { Quality of Life } \\
\text { Questionnaire C30 } \\
\text { (EORTC-CLC-C30) } \\
\text { - } \quad \text { QuickPIPER } \\
\text { C-reactive protein and } \\
\text { creatine kinase levels } \\
\text { (objective measurement of } \\
\text { fatigue and insomnia) } \\
\text { - } \quad \text { Visual analogue scale }\end{array}$ \\
\hline
\end{tabular}


Table 3. Cont

\begin{tabular}{|c|c|c|c|c|c|c|}
\hline $\begin{array}{l}\text { Author/Year/ } \\
\text { Country }\end{array}$ & Settings & Participants/Sample Size & $\begin{array}{l}\text { Intervention } \\
\text { Type }\end{array}$ & Intervention & $\begin{array}{c}\text { Assessed Outcomes of } \\
\text { Interest/Data Collection Time } \\
\text { Points } \\
\end{array}$ & $\begin{array}{l}\text { Instruments for Outcome } \\
\text { Assessments }\end{array}$ \\
\hline $\begin{array}{l}\text { Henry et al., } \\
2018 \text { [34]; USA }\end{array}$ & Not specified & 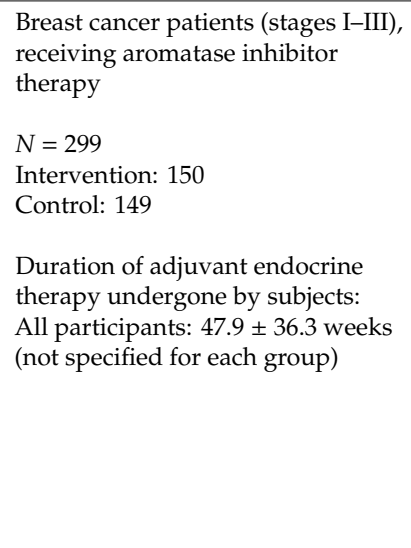 & $\begin{array}{l}\text { Pharmacological } \\
\text { intervention }\end{array}$ & $\begin{array}{l}\text { Pharmacological intervention with } \\
\text { Duloxetine } \\
\text { Intervention: } \\
\text { Intake of } 1 \text { capsule containing } 30 \mathrm{mg} \\
\text { duloxetine per day for } 1 \text { week. Intake of } \\
2 \text { of the capsule described above for the } \\
\text { subsequent } 11 \text { weeks. Thereafter, } \\
\text { patients took } 1 \text { capsule daily for one } \\
\text { further week. } \\
\text { Control: } \\
\text { Intake of placebo containing sugar } \\
\text { spheres over the schedule described } \\
\text { above }\end{array}$ & $\begin{array}{l}\text { - Joint pain and stiffness } \\
\text { - } \quad \text { QOL } \\
\text { Depression } \\
\text { Data collected at: } \\
\text { - } \quad \text { Baseline } \\
\text { - Week } 2 \\
\text { - } \quad \text { Wost-randomization (T1) } \\
\text { - } \quad \text { post-randomization (T2) } \\
\text { Week } 12 \\
\text { - } \quad \text { Wost-randomization (T3) } \\
\quad \text { post-randomization (T4) }\end{array}$ & $\begin{array}{ll}\text { - } & \text { Brief Pain Inventory (BPI) } \\
\text { Western Ontario and } \\
\text { McMaster Universities } \\
\text { Osteoarthritis scale } \\
\text { - } \quad \text { The Global Rating of } \\
\text { Change Scale (GRCS) } \\
\text { - } \quad \text { The Functional } \\
\text { Assessment of Cancer } \\
\text { Therapy-Endocrine Scale } \\
\text { Trial Outcome Index } \\
\text { The brief Patient } \\
\text { Health Questionnaire-9 }\end{array}$ \\
\hline $\begin{array}{l}\text { Hershman et al., } \\
2018 \text { [40]; USA }\end{array}$ & $\begin{array}{l}11 \text { Academic } \\
\text { and } \\
\text { community } \\
\text { sites-sites } \\
\text { not specified }\end{array}$ & $\begin{array}{l}\text { Breast cancer patients, undergoing } \\
\text { aromatase inhibitor therapy } \\
N=226 \\
\text { Intervention: } \\
\text { True acupuncture = } 110 \\
\text { Control Groups: } \\
\text { Sham Acupuncture = 59 } \\
\text { Waiting list = 57 } \\
\text { Duration of adjuvant endocrine } \\
\text { therapy undergone by subjects: } \\
\text { All participants: At least } 1 \text { month } \\
\text { (not specified for each group) }\end{array}$ & $\begin{array}{l}\text { Acupuncture } \\
\text { intervention }\end{array}$ & $\begin{array}{l}\text { Acupuncture intervention } \\
\text { Intervention Group: } \\
12 \text { sessions of true acupuncture over the } \\
\text { first } 6 \text { weeks. Each session lasted for } \\
30-45 \text { min. Six more weekly sessions of } \\
\text { true acupuncture were held over the } \\
\text { next } 6 \text { weeks. } \\
\text { Control Group 1: Sham Acupuncture } \\
\text { Sessions of sham acupuncture based on } \\
\text { the schedule presented above. } \\
\text { Control Group 2: The waitlist control } \\
\text { group } \\
\text { Delayed treatment using true } \\
\text { acupuncture received by intervention } \\
\text { group }\end{array}$ & $\begin{array}{ll}\text { - } & \text { Joint pain and } \\
\text { - } & \text { joint stiffness } \\
\text { - } & \text { Disual functioss about } \\
& \text { sexual problems } \\
\text { Data collected at } & \\
\text { - } & \text { Baseline } \\
\text { - } & 6 \text { weeks after } \\
\text { - } & \text { randomization (T1) } \\
\text { - } & \text { reeks after } \\
\text { - } & 16 \text { weekization (T2) } \\
& \text { randomization (T3) } \\
\text { - } & 20 \text { weeks after } \\
& \text { randomization (T4) } \\
\text { - } & 24 \text { weeks after } \\
& \text { randomization (T5) } \\
\text { - } & 52 \text { weeks after } \\
\text { randomization (T6) }\end{array}$ & $\begin{array}{ll}\text { - The Brief Pain Inventory } \\
\text { Short Form } \\
\text { - Western Ontario and } \\
\text { McMaster Universities } \\
\text { Osteoarthritis Index } \\
\text { - Functional assessment of } \\
\text { Cancer } \\
\text { Therapy-Endocrine Symptoms } \\
\text { - The Patient-Reported } \\
\text { Outcomes Measurement } \\
\text { Information System } \\
\text { (PROMIS) Pain } \\
\text { Impact-Short Form }\end{array}$ \\
\hline
\end{tabular}


Table 3. Cont

\begin{tabular}{|c|c|c|c|c|c|c|}
\hline $\begin{array}{l}\text { Author/Year/ } \\
\text { Country }\end{array}$ & Settings & Participants/Sample Size & $\begin{array}{l}\text { Intervention } \\
\text { Type }\end{array}$ & Intervention & $\begin{array}{c}\text { Assessed Outcomes of } \\
\text { Interest/Data Collection Time } \\
\text { Points }\end{array}$ & $\begin{array}{l}\text { Instruments for Outcome } \\
\text { Assessments }\end{array}$ \\
\hline $\begin{array}{l}\text { Advani et al., } \\
2017 \text { [33]; USA }\end{array}$ & Not specified & $\begin{array}{l}\begin{array}{l}\text { Localized breast cancer patients } \\
\text { (early stage), receiving adjuvant } \\
\text { endocrine therapy with aromatase } \\
\text { inhibitor }\end{array} \\
N=57 \\
\text { Control group: } \\
\text { Usual Care Group: } 21 \\
\text { Intervention groups: } \\
\text { Active Group-H:18 } \\
\text { Active Group-P:18 } \\
\text { Duration of adjuvant endocrine } \\
\text { therapy undergone by subjects: } \\
\text { All participants: Less than } 4 \text { weeks } \\
\text { (not specified for each group) }\end{array}$ & $\begin{array}{l}\text { Multimodal } \\
\text { intervention }\end{array}$ & $\begin{array}{l}\text { A multimodal intervention involving } \\
\text { sexual counseling, and use of vaginal } \\
\text { moisturizers, lubricants, and/or dilator } \\
\text { - A booklet, Why It Is Important to } \\
\text { Take Your Aromatase Inhibitor, } \\
\text { was given to both intervention and } \\
\text { control participants in the study, } \\
\text { encouraging adherence to } \\
\text { endocrine therapy and provision of } \\
\text { information on self-help strategies } \\
\text { for symptom management. } \\
\text { Intervention: } \\
\text { - Provision of one brand of vaginal } \\
\text { moisturizer, each getting a } \\
\text { 6-month supply. } \\
\text { Active Group-H: received an } \\
\text { over-the-counter product } \\
\text { containing a form of } \\
\text { hyaluronic acid. } \\
\text { Active Group-P: received an } \\
\text { over-the-counter moisturizer } \\
\text { labeled as prebiotic to promote } \\
\text { healthy lactobacilli. } \\
\text { Application of vaginal moisturizer } \\
\text { was performed daily during Week } \\
\text { 1, and then 2-3 times } \\
\text { weekly thereafter. } \\
\text { Provision of water-based lubricants } \\
\text { and/or silicone vaginal dilator for } \\
\text { application during sexual activity. }\end{array}$ & $\begin{array}{l}\text { - } \quad \text { Sexual function } \\
\text { Distress about } \\
\text { sexual problems } \\
\text { Dyspareunia } \\
\text { Data collected at: } \\
\text { - } \quad \text { Baseline } \\
\text { - } 6 \text { months after } \\
\text { randomization (T1) } \\
\text { - } 12 \text { months after } \\
\text { randomization (T2) }\end{array}$ & $\begin{array}{ll}\text { - } & \text { Female Sexual Function } \\
& \text { Index (FSFI) } \\
\text { - } & \text { Menopausal Sexual } \\
& \text { Interest Questionnaire } \\
\text { - } & \text { Female Sexual } \\
& \text { Distress Scale-Revised } \\
\text { - } & \text { Breast Cancer Prevention } \\
& \text { Trial Symptom Scale }\end{array}$ \\
\hline & & & & $\begin{array}{l}\text { Control: } \\
\text { Usual Care }\end{array}$ & & \\
\hline
\end{tabular}


Table 3. Cont

\begin{tabular}{|c|c|c|c|c|c|c|}
\hline $\begin{array}{l}\text { Author/Year/ } \\
\text { Country }\end{array}$ & Settings & Participants/Sample Size & $\begin{array}{l}\text { Intervention } \\
\text { Type }\end{array}$ & Intervention & $\begin{array}{c}\text { Assessed Outcomes of } \\
\text { Interest/Data Collection Time } \\
\text { Points }\end{array}$ & $\begin{array}{l}\text { Instruments for Outcome } \\
\text { Assessments }\end{array}$ \\
\hline $\begin{array}{l}\text { Nyrop et al., } \\
2017 \text { [35]; USA }\end{array}$ & $\begin{array}{l}\text { Participants' } \\
\text { home }\end{array}$ & $\begin{array}{l}\text { Breast cancer survivors previously } \\
\text { diagnosed with stage } 0 \text {-III cancer, } \\
\text { undergoing aromatase inhibitor } \\
\text { therapy } \\
N=62 \\
\text { Intervention: } 31 \\
\text { Control: } 31 \\
\text { Duration of adjuvant endocrine } \\
\text { therapy undergone by subjects: } \\
\text { All participants: } 1.7 \pm 1.43 \text { years (not } \\
\text { specified for each group) }\end{array}$ & $\begin{array}{l}\text { Physical activity } \\
\text { intervention }\end{array}$ & $\begin{array}{l}\text { Home-based walking program } \\
\text { Intervention: } \\
\text { - } \quad \begin{array}{l}\text { Participation in a 6-week walking } \\
\text { program where participants were } \\
\text { encouraged to reach the target of } \\
\text { having walked } 150 \text { min per week. } \\
\text { Provision of a brochure with topics } \\
\text { on the importance of physical } \\
\text { activities (walking) and symptoms } \\
\text { associated with cancer treatment } \\
\text { (joint pain). } \\
\text { Provision of activity log to record } \\
\text { physical activity level daily. } \\
\text { Control: } \\
\text { Wait-list control, receiving intervention } \\
\text { after post-intervention data collection }\end{array}\end{array}$ & $\begin{array}{l}\text { - } \text { Joint symptoms } \\
\text { - } \quad \text { Pain } \\
\text { - } \quad \text { Fatigue } \\
\text { Data collected at: } \\
\text { - } \quad \text { Baseline } \\
\text { - } \quad \text { post-intervention (T1)—6 } \\
\text { - } \quad 6 \text { moeks follow-up } \\
\text { post-intervention (T2) }\end{array}$ & $\begin{array}{ll}\text { - } & \text { Visual Analog Scale (VAS) } \\
\text { Western Ontario and } \\
\text { McMaster Universities } \\
\text { Arthritis Index } \\
\text { - Functional Assessment of } \\
\text { Cancer Therapy-General } \\
\text { (FACT-G) }\end{array}$ \\
\hline $\begin{array}{l}\text { Rogers et al., } \\
2017 \text { [48]; USA }\end{array}$ & $\begin{array}{l}\text { Local } \\
\text { institutions, } \\
\text { with } \\
\text { home-based } \\
\text { exercises }\end{array}$ & $\begin{array}{l}\text { Breast cancer survivors (stages } \\
\text { I-IIIA), receiving hormonal therapy } \\
N=222 \\
\text { Intervention: } 110 \\
\text { Control: } 112 \\
\text { Duration of adjuvant endocrine } \\
\text { therapy undergone by subjects: } \\
\text { Not specified }\end{array}$ & $\begin{array}{l}\text { Physical activity } \\
\text { intervention }\end{array}$ & $\begin{array}{l}\text { Physical activity behavior change } \\
\text { intervention (Better Exercise Adherence } \\
\text { after Treatment for Cancer) } \\
\text { Intervention: } \\
\text { - Attendance to } 12 \text { supervised } \\
\text { exercise sessions for } 6 \text { weeks, } \\
\text { supplemented by unsupervised } \\
\text { home-based exercises. } \\
\text { Attendance to counselling sessions } \\
\text { with exercise specialists every } \\
2 \text { weeks. } \\
\text { Attendance to } 6 \text { group discussion } \\
\text { sessions on topics including the } \\
\text { benefits of and barriers to doing } \\
\text { exercises, goal setting for } \\
\text { exercise levels. } \\
\text { Control: } \\
\text { Usual care }\end{array}$ & $\begin{array}{l}\text { - } \quad \text { Sleep quality } \\
\text { Data collected at: } \\
\text { - } \quad \text { Baseline } \\
\text { - } \quad \text { Immediately } \\
\text { post-intervention (T1) } \\
\text { - } 3 \text { months } \\
\text { post-intervention (T2) }\end{array}$ & $\begin{array}{l}\text { - Pittsburgh Sleep } \\
\text { Quality Index }\end{array}$ \\
\hline
\end{tabular}


Table 3. Cont.

\begin{tabular}{|c|c|c|c|c|c|c|}
\hline $\begin{array}{l}\text { Author/Year/ } \\
\text { Country }\end{array}$ & Settings & Participants/Sample Size & $\begin{array}{l}\text { Intervention } \\
\text { Type }\end{array}$ & Intervention & $\begin{array}{c}\text { Assessed Outcomes of } \\
\text { Interest/Data Collection Time } \\
\text { Points }\end{array}$ & $\begin{array}{l}\text { Instruments for Outcome } \\
\text { Assessments }\end{array}$ \\
\hline $\begin{array}{l}\text { Hershman et al., } \\
2015 \text { [39]; USA }\end{array}$ & Not specified & $\begin{array}{l}\text { Breast cancer survivors (stages I-III), } \\
\text { receiving adjuvant aromatase } \\
\text { inhibitor therapy } \\
N=262 \\
\text { Intervention: } 131 \\
\text { Control: } 131 \\
\text { Duration of adjuvant endocrine } \\
\text { therapy undergone by subjects: } \\
\text { Intervention group: } 1.1 \text { years } \\
\text { (median) } \\
\text { Control group: } 1.3 \text { years (median) }\end{array}$ & $\begin{array}{l}\text { Dietary } \\
\text { intervention }\end{array}$ & $\begin{array}{l}\text { Dietary intervention with omega- } 3 \text { fatty } \\
\text { acids } \\
\text { Intervention: } \\
\text { Intake of } 6 \text { capsules containing } 3.3 \mathrm{~g} \\
\text { omega- } 3 \text { fatty acid (eicosapentaenoic } \\
\text { acid and docosahexaenoic acid) per day, } \\
\text { over a period of } 24 \text { weeks. } \\
\text { Control: } \\
\text { Intake of placebo capsule containing } \\
\text { soybean oil and corn oil, } 6 \text { per day over } \\
24 \text { weeks. }\end{array}$ & $\begin{array}{l}\text { - Joint symptoms (pain } \\
\text { and stiffness) } \\
\text { - } \quad \text { Functional status } \\
\text { Data collected at: } \\
\text { - Baseline } \\
\text { - Week } 6 \text { of intervention } \\
\text { - Week } 12 \text { of intervention } \\
\quad \begin{array}{l}\text { (immeek } 24 \text { of intervention } \\
\text { intervention) }\end{array}\end{array}$ & $\begin{array}{ll}- & \text { Brief Pain Inventory (BPI) } \\
\text { - } & \text { Western Ontario and } \\
\text { McMaster Universities } \\
\text { Osteoarthritis Index } \\
\text { (WOMAC) } \\
\text { - } \quad \text { Modified Score for the } \\
\text { Assessment and } \\
\text { Quantification of Chronic } \\
\text { Rheumatoid Affections of } \\
\text { the Hands (MSACRAH) } \\
\text { Serum C-reactive protein } \\
\text { (CRP) levels (objective } \\
\text { measure of pain) }\end{array}$ \\
\hline $\begin{array}{l}\text { Irwin et al., } 2015 \\
\text { [38], USA }\end{array}$ & $\begin{array}{l}\text { Local health } \\
\text { club and } \\
\text { home-based }\end{array}$ & $\begin{array}{l}\text { Breast cancer Survivors (stages I-III), } \\
\text { undergoing aromatase inhibitor } \\
\text { therapy } \\
N=121 \\
\text { Intervention group = } 61 \\
\text { Control Group = } 60 \\
\text { Duration of adjuvant endocrine } \\
\text { therapy undergone by subjects: } \\
\text { Intervention group: } 1.9 \pm 2.9 \text { years } \\
\text { Control group: } 1.8 \pm 1.3 \text { years }\end{array}$ & $\begin{array}{l}\text { Physical activity } \\
\text { intervention }\end{array}$ & $\begin{array}{l}\text { Year-long exercise intervention } \\
\text { Intervention Group: } \\
\text { Participation in a supervised resistance } \\
\text { exercise program with sessions held } \\
\text { twice a week, and a home-based aerobic } \\
\text { exercise program (brisk walking or } \\
\text { stationary cycling) of } 150 \text { min per week. } \\
\text { The program lasted for } 1 \text { year. } \\
\text { Control Group } \\
\text { Usual care } \\
\text { Participants in both groups were given } \\
\text { education booklets with information on } \\
\text { breast cancer-related symptoms } \\
\text { including lymphedema and fatigue }\end{array}$ & $\begin{array}{l}\text { - } \quad \text { Joint pain } \\
\text { Data collected at } \\
\text { - } \quad \text { Baseline } \\
\text { - } 3 \text { months after } \\
\text { randomization (T1) } \\
\text { - } 6 \text { months after } \\
\text { randomization (T2) } \\
\text { - } 9 \text { months after } \\
\text { randomization (T3) } \\
\text { - } 12 \text { months after } \\
\text { randomization (T4) }\end{array}$ & $\begin{array}{ll}- & \text { Brief Pain Inventory } \\
\text { - } & \text { Western Ontario and } \\
\text { McMaster Universities } \\
\text { Osteoarthritis Index } \\
\text { (WOMAC) }\end{array}$ \\
\hline
\end{tabular}


Table 3. Cont

\begin{tabular}{|c|c|c|c|c|c|c|}
\hline $\begin{array}{l}\text { Author/Year/ } \\
\text { Country }\end{array}$ & Settings & Participants/Sample Size & $\begin{array}{l}\text { Intervention } \\
\text { Type }\end{array}$ & Intervention & $\begin{array}{c}\text { Assessed Outcomes of } \\
\text { Interest/Data Collection Time } \\
\text { Points }\end{array}$ & $\begin{array}{l}\text { Instruments for Outcome } \\
\text { Assessments }\end{array}$ \\
\hline $\begin{array}{l}\text { Peppone et al., } \\
2015 \text { [45]; USA }\end{array}$ & $\begin{array}{l}\text { Community-basec } \\
\text { and } \\
\text { group-based }\end{array}$ & $\begin{array}{l}\text { Breast cancer survivors (stages } 0 \text {-III) } \\
\text { receiving either tamoxifen or } \\
\text { aromatase inhibitor therapy } \\
N=167 \\
\text { Intervention: } 75 \\
\text { Control: } 92 \\
\text { Duration of adjuvant endocrine } \\
\text { therapy undergone by subjects: } \\
\text { Not specified }\end{array}$ & $\begin{array}{l}\text { Psychotherapeutic } \\
\text { intervention }\end{array}$ & $\begin{array}{l}\text { Yoga intervention } \\
\text { Intervention: } \\
\text { Attendance to group sessions of yoga, } \\
\text { involving breathing and mindfulness } \\
\text { exercises and physical alignment } \\
\text { postures. Each session lasted } 75 \text { min, } \\
\text { and was held twice a week over a period } \\
\text { of } 4 \text { weeks. } \\
\text { Control: } \\
\text { Wait-list control, receiving standard care } \\
\text { during the intervention period. }\end{array}$ & $\begin{array}{l}\text { - } \quad \text { Fatigue } \\
\text { Data collected at: } \\
\text { - } \quad \text { Baseline } \\
\text { - Immediately post- } \\
\text { intervention }\end{array}$ & $\begin{array}{l}\text { Selected items from: } \\
\text { - } \quad \text { University of Rochester } \\
\text { Cancer Center } \\
\text { Symptom Inventory } \\
\text { - } \quad \text { Functional Assessment of } \\
\text { Chronic Illness Therapy } \\
\text { with Fatigue Subscale } \\
\text { (FACIT-F) } \\
\text { Multidimensional Fatigue } \\
\text { Symptom Inventory-Short } \\
\text { Form (MFSI-SF) }\end{array}$ \\
\hline $\begin{array}{l}\text { Mao et al., } 2014 \\
\text { [46], USA }\end{array}$ & $\begin{array}{l}\text { Tertiary care } \\
\text { academic } \\
\text { medical center }\end{array}$ & $\begin{array}{l}\text { Breast cancer patients (stages I-III), } \\
\text { currently undergoing aromatase } \\
\text { inhibitor therapy } \\
N=67 \\
\text { Intervention }=22 \\
\text { Sham acupuncture control } \\
\text { group }=22 \\
\text { Waitlist Control }=23 \\
\\
\text { Duration of adjuvant endocrine } \\
\text { therapy undergone by subjects: } \\
\text { Intervention group: } 26.9 \pm 17.3 \\
\text { months } \\
\text { Sham acupuncture control group: } \\
19.5 \pm 16.9 \text { months } \\
\text { Control group: } 31.1 \pm 22.1 \text { months }\end{array}$ & $\begin{array}{l}\text { Acupuncture } \\
\text { intervention }\end{array}$ & $\begin{array}{l}\text { Electroacupuncture (EA) intervention } \\
\text { Intervention } \\
\text { Undertake } 30 \text {-min electroacupuncture } \\
\text { sessions, held twice a week for } 2 \text { weeks, } \\
\text { then weekly for } 6 \text { more weeks } \\
\text { Sham Electroacupuncture (SA) } \\
\text { Undertake 30-min electroacupuncture } \\
\text { sessions, held twice a week for } 2 \text { weeks, } \\
\text { then weekly for } 6 \text { more weeks, but with } \\
\text { non-penetrating needles. } \\
\text { Waitlist Control } \\
\text { Usual care }\end{array}$ & 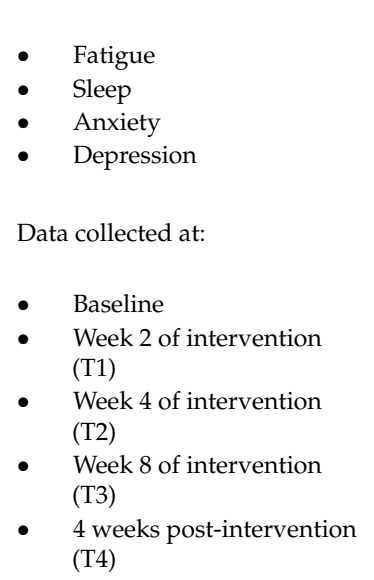 & $\begin{array}{ll}\text { - } & \text { Brief Fatigue Inventory } \\
& \text { (BFI) } \\
\text { - } & \text { Pittsburgh Sleep Quality } \\
& \text { Index (PSQI) } \\
\text { - } & \text { Hospital Anxiety and } \\
& \text { Depression Scale (HADS) }\end{array}$ \\
\hline
\end{tabular}


Table 3. Cont.

\begin{tabular}{|c|c|c|c|c|c|c|}
\hline $\begin{array}{l}\text { Author/Year/ } \\
\text { Country }\end{array}$ & Settings & Participants/Sample Size & $\begin{array}{l}\text { Intervention } \\
\text { Type }\end{array}$ & Intervention & $\begin{array}{c}\text { Assessed Outcomes of } \\
\text { Interest/Data Collection Time } \\
\text { Points }\end{array}$ & $\begin{array}{l}\text { Instruments for Outcome } \\
\text { Assessments }\end{array}$ \\
\hline $\begin{array}{l}\text { Oh et al., } 2013 \\
\text { [41], Australia }\end{array}$ & $\begin{array}{l}\text { Tertiary } \\
\text { Teaching } \\
\text { Hospital }\end{array}$ & 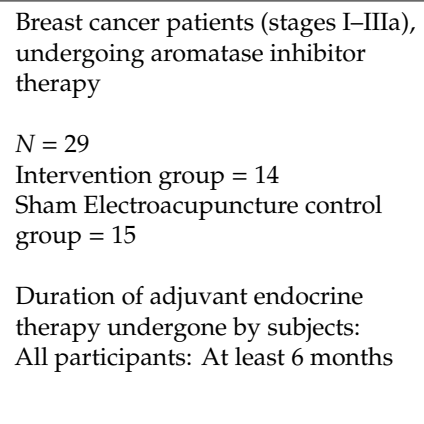 & $\begin{array}{l}\text { Acupuncture } \\
\text { intervention }\end{array}$ & $\begin{array}{l}\text { Electroacupuncture (EA) intervention } \\
\text { Intervention Group: } \\
\text { Undertake 20-min sessions of real } \\
\text { electroacupuncture twice weekly for } 6 \\
\text { weeks, using acupuncture needles } \\
\text { Control Group: } \\
\text { Undertake 20-min sessions of sham } \\
\text { electroacupuncture twice weekly for } 6 \\
\text { weeks, using sham acupuncture needles } \\
\text { that do not penetrate the skin }\end{array}$ & $\begin{array}{l}\text { - Joint pain and } \\
\text { - } \quad \text { QOint stiffness } \\
\text { Data collected at: } \\
\text { - Baseline } \\
\text { - } \quad \text { Immediate } \\
\text { - } \quad \text { 6-most-intervention (T1) } \\
\text { post-intervention (T2) }\end{array}$ & $\begin{array}{ll}\text { - } & \text { Brief Pain Inventory } \\
\text { - } & \text { Whort Form } \\
\text { Mcstern Ontario and } \\
\text { Osteoarthritis Index } \\
\text { - } \quad \text { Functional assessment of } \\
\text { Cancer } \\
\text { Therapy-General Symptoms }\end{array}$ \\
\hline $\begin{array}{l}\text { Mann et al., } 2012 \\
\text { [30]; United } \\
\text { Kingdom }\end{array}$ & Not specified & 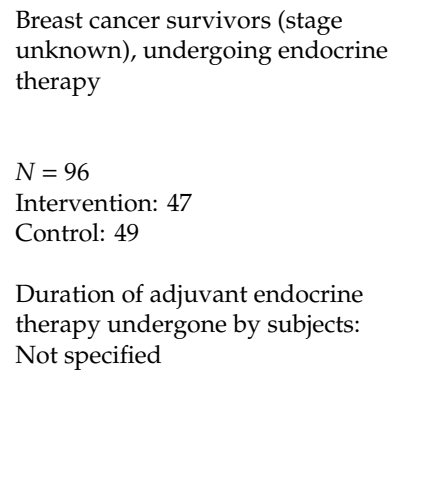 & $\begin{array}{l}\text { Psychotherapeutic } \\
\text { intervention }\end{array}$ & $\begin{array}{l}\text { Cognitive behavioral therapy (CBT) } \\
\text { Intervention: } \\
\text { Attendance to six 90-min group-based } \\
\text { CBT sessions, held once a week, over a } \\
\text { period of } 6 \text { weeks. These sessions } \\
\text { included the provision of } \\
\text { psycho-education via presentations and } \\
\text { handouts, group discussions and } \\
\text { homework assignments. } \\
\text { Control: } \\
\text { Usual care }\end{array}$ & $\begin{array}{l}\text { - } \quad \text { Perceived burden of hot } \\
\text { - } \quad \text { Emotion and night sweats } \\
\text { - } \quad \text { Sleep problems } \\
\text { - } \quad \text { RRQOL } \\
\text { Data collected at: } \\
\text { - } \quad \text { Baseline } \\
\text { - } 9 \text { weeks after } \\
\text { - } \quad 26 \text { weeks after } \\
\text { randomization (T2) }\end{array}$ & $\begin{array}{ll}\text { - } & \text { Hot Flush Rating Scale } \\
\text { - } & \text { Women's } \\
\text { - } & \text { Health Questionnaire } \\
& \text { Seneral Health Survey } \\
& \text { Short Form } 36\end{array}$ \\
\hline
\end{tabular}


Table 3. Cont

\begin{tabular}{|c|c|c|c|c|c|c|}
\hline $\begin{array}{l}\text { Author/Year/ } \\
\text { Country }\end{array}$ & Settings & Participants/Sample Size & $\begin{array}{l}\text { Intervention } \\
\text { Type }\end{array}$ & Intervention & $\begin{array}{c}\text { Assessed Outcomes of } \\
\text { Interest/Data Collection Time } \\
\text { Points }\end{array}$ & $\begin{array}{l}\text { Instruments for Outcome } \\
\text { Assessments }\end{array}$ \\
\hline $\begin{array}{l}\text { Zhao et al., } 2012 \\
\text { [44]; China }\end{array}$ & Not specified & $\begin{array}{l}\text { Breast cancer survivors previously } \\
\text { diagnosed with stage I-IIIA cancer, } \\
\text { having completed or undergoing } \\
\text { endocrine therapy } \\
\begin{array}{l}N=48 \\
\text { Intervention: } 25 \\
\text { Control: } 23 \\
\text { Duration of adjuvant endocrine } \\
\text { therapy undergone by subjects: } \\
\text { All participants: } 6 \text { months-5 years } \\
\text { (not specified for each group) }\end{array}\end{array}$ & $\begin{array}{l}\text { Pharmacological } \\
\text { intervention }\end{array}$ & $\begin{array}{l}\text { Pharmacological intervention with spore } \\
\text { powder of G. lucidum } \\
\text { Intervention: } \\
\text { Intake of } 1000 \mathrm{mg} \text { spore powder of } G \text {. } \\
\text { lucidum three times a day for } 4 \text { weeks } \\
\text { Control: } \\
\text { Intake of placebo, three times a day for } 4 \\
\text { weeks }\end{array}$ & $\begin{array}{l}\text { - Fatigue } \\
\text { - } \text { QOLxiety and depression } \\
\text { Data collected at: } \\
\text { - Baseline } \\
\text { - Post-intervention }\end{array}$ & $\begin{array}{l}\text { - Functional Assessment of } \\
\text { Cancer Therapy: Fatigue } \\
\text { (FACT-F) } \\
\text { - The Hospital Anxiety and } \\
\text { Depression Scale } \\
\text { - European Organization } \\
\text { for Research and } \\
\text { Treatment of Cancer Core } \\
\text { Quality of Life } \\
\text { Questionnaire C30 } \\
\text { (EORTC-CLC-C30) } \\
\text { Seral level of TNF- } \alpha \text { and } \\
\text { IL-6 (objective } \\
\text { measurement of fatigue) }\end{array}$ \\
\hline $\begin{array}{l}\text { Rogers et al., } \\
2009 \text { [36]; USA }\end{array}$ & Not specified & $\begin{array}{l}\text { Breast cancer survivors (stages } \\
\text { I-IIIA), currently on aromatase } \\
\text { inhibitor therapy or estrogen } \\
\text { receptor modulator therapy } \\
N=41 \\
\text { Intervention: } 21 \\
\text { Control: } 20 \\
\text { Duration of adjuvant endocrine } \\
\text { therapy undergone by subjects: } \\
\text { All participants: } 18 \pm 17 \text { months (not } \\
\text { specified for each group) }\end{array}$ & $\begin{array}{l}\text { Physical activity } \\
\text { intervention }\end{array}$ & $\begin{array}{l}\text { Physical activity behavior change } \\
\text { intervention (The BEAT Cancer } \\
\text { Program) } \\
\text { Intervention: } \\
\text { - During the 12-week intervention, } \\
\text { participants attended } 12 \text { individual } \\
\text { supervised exercise sessions for the } \\
\text { first } 6 \text { weeks and } 3 \text { counselling } \\
\text { sessions face-to-face with an } \\
\text { exercise specialist for the next } 6 \\
\text { weeks to tailor a home-based } \\
\text { exercise program at the end of the } \\
\text { intervention and enhance the } \\
\text { ability of participants to } \\
\text { self-monitor their physical } \\
\text { activity level } \\
\text { Participation in } 6 \text { group discussion } \\
\text { sessions focusing on journaling, } \\
\text { time and stress management, } \\
\text { barriers to exercise and } \\
\text { behavioral change } \\
\text { Control: } \\
\text { Usual care }\end{array}$ & $\begin{array}{l}\text { - } \quad \text { QOL } \\
\text { - } \quad \text { Sleep quality } \\
\text { Data collected at: } \\
\text { - } \quad \text { Baseline } \\
\text { - } \quad \text { Immediately } \\
\text { post-intervention (T1) } \\
\text { - } 3 \text { months } \\
\quad \text { post-intervention (T2) }\end{array}$ & $\begin{array}{ll}\text { - } & \text { Functional Assessment of } \\
\text { Cancer Therapy-Breast } \\
\text { (FACT-B) } \\
\text { - } \quad \text { Pittsburgh Sleep } \\
\text { Quality Index } \\
\text { - Western Ontario and } \\
\text { McMaster Universities } \\
\text { Osteoarthritis Index }\end{array}$ \\
\hline
\end{tabular}


Table 3. Cont.

\begin{tabular}{|c|c|c|c|c|c|c|}
\hline $\begin{array}{l}\text { Author/Year/ } \\
\text { Country }\end{array}$ & Settings & Participants/Sample Size & $\begin{array}{l}\text { Intervention } \\
\text { Type }\end{array}$ & Intervention & $\begin{array}{c}\text { Assessed Outcomes of } \\
\text { Interest/Data Collection Time } \\
\text { Points }\end{array}$ & $\begin{array}{l}\text { Instruments for Outcome } \\
\text { Assessments }\end{array}$ \\
\hline $\begin{array}{l}\text { Rogers et al., } \\
2009 \text { [37]; USA }\end{array}$ & Not specified & Same as Rogers et al, 2009 [36] & $\begin{array}{l}\text { Physical activity } \\
\text { intervention }\end{array}$ & Same as Rogers et al., 2009 [36] & $\begin{array}{l}\text { - } \quad \text { QOL } \\
\text { - } \quad \text { Sleep quality } \\
\text { Data collected at: } \\
\text { - } \quad \text { Baseline } \\
\text { - } \quad \text { Immediately post- } \\
\quad \text { intervention }\end{array}$ & $\begin{array}{ll}\text { - } & \text { Functional Assessment of } \\
\text { Cancer Therapy-Breast } \\
\text { (FACT-B) } \\
\text { - Pittsburgh Sleep Quality } \\
\text { Index (PSQI) } \\
\text { - } \\
\text { Western Ontario and } \\
\text { McMaster Universities } \\
\text { Osteoarthritis Index }\end{array}$ \\
\hline $\begin{array}{l}\text { Crew et al., } 2007 \\
\text { [42]; USA }\end{array}$ & Not specified & $\begin{array}{l}\text { Breast cancer survivors (stages } \\
\text { I-IIIa), undertaking aromatase } \\
\text { inhibitor therapy } \\
\begin{array}{l}N=21 \\
\text { Intervention: Not mentioned } \\
\text { Control: Not mentioned } \\
\text { Duration of adjuvant endocrine } \\
\text { therapy undergone by subjects: } \\
\text { All participants: At least } 6 \text { months } \\
\text { (not specified for each group) }\end{array}\end{array}$ & $\begin{array}{l}\text { Acupuncture } \\
\text { intervention }\end{array}$ & $\begin{array}{l}\text { Acupuncture intervention } \\
\text { Intervention: } \\
\text { Acupuncture session lasting } 30 \mathrm{~min} \text {, } \\
\text { performed two times a week for six } \\
\text { weeks. } \\
\text { Control: } \\
\text { Wait-list control }\end{array}$ & $\begin{array}{l}\text { - Joint pain and stiffness } \\
\text { - } \quad \text { QOL } \\
\text { inflammatory markers, } \\
\text { IL-1 } \beta \text { and TNF- } \alpha \\
\text { Data collected at: } \\
\text { - } \quad \text { Baseline } \\
\text { - } \quad 6 \text { weeks after } \\
\text { randomization (T1) } \\
\text { - } \quad 12 \text { weeks after } \\
\text { randomization (T2) }\end{array}$ & $\begin{array}{l}\text { - } \quad \text { Brief Pain Inventory-Short } \\
\text { - } \quad \text { Form (BPI-SF) } \\
\text { Western Ontario and } \\
\text { McMaster Universities } \\
\text { Osteoarthritis (WOMAC) index } \\
\text { - } \quad \text { The Functional } \\
\text { Assessment of Cancer } \\
\text { Therapy-General quality } \\
\text { of life measure } \\
\text { - Enzyme-linked } \\
\text { immunosorbent assay } \\
\text { (ELISA) }\end{array}$ \\
\hline $\begin{array}{l}\text { Kimmick et al., } \\
2006 \text { [27]; USA }\end{array}$ & Not specified & $\begin{array}{l}\text { Breast cancer survivors (stages } \\
0 \text {-IIIB), receiving tamoxifen therapy } \\
N=62 \\
\text { Intervention: } 33 \\
\text { Control: } 29 \\
\text { Duration of adjuvant endocrine } \\
\text { therapy undergone by subjects: } \\
\text { Not specified }\end{array}$ & $\begin{array}{l}\text { Pharmacological } \\
\text { intervention } \\
\text { (a crossover } \\
\text { trial) }\end{array}$ & $\begin{array}{l}\text { Pharmacological intervention with the } \\
\text { antidepressant sertraline } \\
\text { Intervention: } \\
\text { Intake of } 50 \mathrm{mg} \text { sertraline per day, over a } \\
\text { period of } 6 \text { weeks. Intervention } \\
\text { participants then took the placebo } \\
\text { tablets per day for the next } 6 \text { weeks. } \\
\text { Control: } \\
\text { Intake of placebo tablet per day, over a } \\
\text { period of } 6 \text { weeks. Control participants } \\
\text { then took } 50 \mathrm{mg} \text { sertraline per day for } \\
\text { the next } 6 \text { weeks. }\end{array}$ & $\begin{array}{ll}\text { - } & \text { Hot flashes frequency } \\
\text { and severity } \\
\text { - } & \text { Depression } \\
\text { - } & \text { QOL } \\
\text { Data collected at: } \\
\text { - } \quad \text { Baseline } \\
\text { - } \quad \text { weeks after start of } \\
\text { intervention (T1) } \\
\text { - } \quad \text { in weeks after start of } \\
\quad \text { intervention (T2) }\end{array}$ & $\begin{array}{l}\text { - Daily diary for hot flashes } \\
\text { - Center for Epidemiologic } \\
\text { Studies depression } \\
\text { (CESD) } \\
\text { - Functional Assessment of } \\
\text { Cancer Therapy-Breast } \\
\text { (FACT-B) }\end{array}$ \\
\hline
\end{tabular}


Table 3. Cont

\begin{tabular}{|c|c|c|c|c|c|c|}
\hline $\begin{array}{l}\text { Author/Year/ } \\
\text { Country }\end{array}$ & Settings & Participants/Sample Size & $\begin{array}{l}\text { Intervention } \\
\text { Type }\end{array}$ & Intervention & $\begin{array}{c}\text { Assessed Outcomes of } \\
\text { Interest/Data Collection Time } \\
\text { Points }\end{array}$ & $\begin{array}{l}\text { Instruments for Outcome } \\
\text { Assessments }\end{array}$ \\
\hline $\begin{array}{l}\text { Pandya et al., } \\
2000 \text { [28]; USA }\end{array}$ & $\begin{array}{l}\text { University of } \\
\text { Rochester } \\
\text { Cancer Centre }\end{array}$ & $\begin{array}{l}\text { Breast cancer survivors (stage } \\
\text { unknown), receiving adjuvant } \\
\text { tamoxifen therapy } \\
N=198 \\
\text { Intervention: } 99 \\
\text { Control: } 99 \\
\text { Duration of adjuvant endocrine } \\
\text { therapy undergone by subjects: } \\
\text { All participants: At } 1 \text { month (not } \\
\text { specified for each group) }\end{array}$ & $\begin{array}{l}\text { Pharmacological } \\
\text { intervention }\end{array}$ & $\begin{array}{l}\text { Pharmacological intervention with } \\
\text { clonidine } \\
\text { Intervention: } \\
\text { Intake of } 0.1 \mathrm{mg} \text { oral clonidine, once } \\
\text { daily, for a period of } 8 \text { weeks. } \\
\text { Control: } \\
\text { Intake of } 0.1 \mathrm{mg} \text { placebo, once daily, for } \\
\text { a period of } 8 \text { weeks. }\end{array}$ & 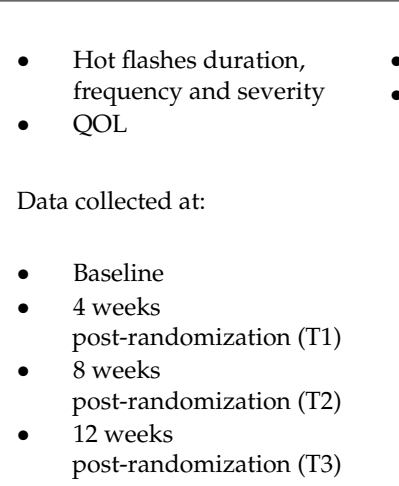 & $\begin{array}{l}\text { - Daily diary for hot flashes } \\
\text { A 10-point rating scale } \\
\text { (for QOL assessment) }\end{array}$ \\
\hline $\begin{array}{l}\text { Goldberg et al., } \\
1994 \text { [29]; USA }\end{array}$ & Not specified & $\begin{array}{l}\text { Breast cancer patients (stage } \\
\text { unknown), receiving tamoxifen } \\
\text { therapy } \\
N=110 \\
\text { Intervention: } 55 \\
\text { Control: } 55 \\
\text { Duration of adjuvant endocrine } \\
\text { therapy undergone by subjects: } \\
\text { Not specified }\end{array}$ & $\begin{array}{l}\text { Pharmacological } \\
\text { intervention }\end{array}$ & $\begin{array}{l}\text { Pharmacological intervention with } \\
\text { transdermal clonidine } \\
\text { Intervention: } \\
\text { Administered transdermal clonidine } \\
\text { patch (equivalent to the oral dose of } 0.1 \\
\text { mg of the drug) daily for a period of } 4 \\
\text { weeks, then intervention participants } \\
\text { were administered the placebo } \\
\text { transdermally daily for the next } 4 \text { weeks. } \\
\text { Control: } \\
\text { Administered the placebo patch to be } \\
\text { used daily for a period of } 4 \text { weeks, then } \\
\text { control participants were administered } \\
\text { the transdermal clonidine patch } \\
\text { (equivalent to the oral dose of } 0.1 \mathrm{mg} \text { of } \\
\text { the drug), used daily for the next } 4 \\
\text { weeks. }\end{array}$ & $\begin{array}{l}\text { - Hot flashes frequency } \\
\text { and severity } \\
\text { Data collected at: } \\
\text { - } \text { Baseline } \\
\text { - Immediately post-intervention } \\
\text { Post-crossover }\end{array}$ & $\begin{array}{l}\text { - Daily } \\
\text { patient questionnaire } \\
\text { on }\end{array}$ \\
\hline
\end{tabular}




\subsubsection{Menopausal Symptoms}

Menopausal symptoms investigated in this review include hot flashes, night sweats and vaginal atrophy. An overview of the intervention effect on menopausal symptoms is provided in Table 4.

\section{Hot Flashes}

Five studies reported the effect of pharmacological interventions on addressing hot flashes among the participants [25-29]. While earlier studies showed that pharmacological interventions using clonidine could lead to significant reduction in hot flashes score, frequency and intensity [28,29], other studies reported non-significant differences $(p \geq 0.54)$ in the changes of these parameters between the intervention and control group at pre- and post-intervention [25-27]. Notably, both the intervention and control participants reported a decrease in these parameters from baseline to post-intervention $[25,26]$, which demonstrates why non-significant differences were observed. Interestingly, in a cross-over study on a pharmacological intervention using the anti-depressant sertraline, significant between-group differences were observed in hot flashes frequency and hot flashes score after cross-over at Week 12 of the intervention, despite the lack of such difference before the cross-over at Week 6 [27]. This suggests that the intervention may take longer for the exhibition of its alleviation effect on hot flashes. These data demonstrate that certain pharmacological products, which have not been intended for use to treat menopausal symptoms, could still be of value for use in pharmacological interventions to alleviate hot flashes among breast cancer patients undergoing adjuvant endocrine therapy.

Moreover, Mann et al. [30] reported the effect of a cognitive behavioral therapy on the perceived burden of hot flashes. The intervention exhibited a significant effect on reducing such burden at Week 9 and Week 26 of the study.

\section{Night Sweats}

Mann et al. [30] also examined the effect of the cognitive behavioral therapy on reducing the perceived burden on night sweats. Similar to hot flashes burden, the intervention exhibited an alleviation effect on perceived night sweats burden at both Week 9 and Week 26 of the study.

\section{Vaginal Atrophy}

Keshavarzi et al. [31] reported the use of a vitamin D/vitamin E vaginal suppository intervention in addressing vaginal atrophy among breast cancer survivors. Compared to controls, patients receiving the intervention had significantly greater decrease in perceived levels of vaginal atrophy, as assessed by the genitourinary atrophy score, throughout the eight-week intervention $(p \leq 0.017)$. No longer-term assessment on patient outcomes was conducted to assess whether there are any long-term effects of the intervention.

\subsubsection{Sexual Dysfunction}

Table 4 presents a summary of the effects of interventions on sexual problems among breast cancer survivors.

De Sousa Vieira et al. [25] reported that a pharmacological intervention using a medicinal plant extract (Guarana) did not have significant effect on increasing sex drive among the participants. Likewise, an exercise training intervention did not exhibit significant enhancement of sexual functioning or sexual enjoyment among participants [32]. A multimodal intervention, comprising sexual counselling and the use of vaginal moisturizers, lubricants and/or dilator, contributed to a greater improvement of sexual function and reduction of sexual distress at post-intervention among intervention participants $(p \leq 0.04)$ [33]. Moreover, intervention participants tended to have a reduced level of dyspareunia compared with control participants at post-intervention $(p=0.07)$. These data indicate that a multimodal intervention combining the delivery of sexual counselling and vaginal pain relief could be considered for use in addressing sexual problems among breast cancer survivors. 
Table 4. A summary of the effects of reported interventions on individual adjuvant endocrine therapy-induced menopausal symptoms and sexual issues.

\begin{tabular}{|c|c|c|c|c|}
\hline $\begin{array}{c}\text { Menopausal } \\
\text { Symptom/Issue }\end{array}$ & Intervention Type & Intervention Name & Major Findings on Intervention Effects on Symptom * & Reference \\
\hline \multirow[t]{4}{*}{ Hot flashes } & \multirow[t]{4}{*}{$\begin{array}{l}\text { Pharmacological } \\
\text { intervention }\end{array}$} & $\begin{array}{l}\text { Pharmacological intervention } \\
\text { with tablets of a medicinal plant } \\
\text { extract (Paullinia cupana or } \\
\text { Guarana) }\end{array}$ & $\begin{array}{l}\text { - No significant difference in the extent of decrease in frequency }(p=0.54) \\
\text { and intensity }(p=0.84) \text { of hot flashes between groups. Both groups } \\
\text { exhibited a decrease in these parameters. }\end{array}$ & $\begin{array}{l}\text { de Sousa } \\
\text { Vieira et al., } \\
2019[25]\end{array}$ \\
\hline & & $\begin{array}{l}\text { Pharmacological intervention } \\
\text { with a homeopathic medicine } \\
\text { named Actheane }{ }^{\circledR}\end{array}$ & $\begin{array}{l}\text { (T1: } 4 \text { weeks after randomization; T2: } 8 \text { weeks after randomization) } \\
\text { - There was no difference in the hot flashes score between the intervention } \\
\text { and control participants at T1 ( } p=0.756) \text {, and T2 }(p=0.775) \text {. Both groups } \\
\text { exhibited a decrease in hot flashes score between baseline and T1/T2. } \\
\text { No significant differences in mean daily hot flashes frequency or intensity } \\
\text { between groups at all data collection time points ( } p \text { values not reported). } \\
\text { - However, at both T1 and T2, the majority of the participants exhibited a } \\
\text { decrease in mean daily hot flashes frequency ( }(71 \% \text { and } 74 \% \text {, respectively), } \\
\text { while some of them had decreased mean daily hot flashes intensity (21\% } \\
\text { and } 27 \% \text {, respectively). } \\
\text { Almost half of the participants expressed that the impact of hot flashes on } \\
\text { their quality of life has been reduced at both T1 }(47 \%) \text { and T2 }(50 \%) \text {. }\end{array}$ & $\begin{array}{l}\text { Heudel et al., } \\
2019 \text { [26] }\end{array}$ \\
\hline & & \multirow[t]{2}{*}{$\begin{array}{l}\text { Pharmacological intervention } \\
\text { with the antidepressant } \\
\text { sertraline }\end{array}$} & $\begin{array}{l}\text { Before cross-over at } 6 \text { weeks after start of intervention (T1) } \\
\text { - No significant between-group differences in the hot flashes frequency } \\
(p=0.80 \text { ) and hot flashes score }(p=0.50) \text {. } \\
\text { No significant difference in the proportion of participants achieving } 50 \% \\
\text { reduction of hot flashes frequency between intervention and control } \\
\text { groups ( } 36 \% \text { vs. } 27 \% ; p=0.70) \text {. }\end{array}$ & \multirow[t]{2}{*}{$\begin{array}{l}\text { Kimmick et al. } \\
2006 \text { [27] }\end{array}$} \\
\hline & & & $\begin{array}{l}\text { After cross-over at } 12 \text { weeks after start of intervention (T2) } \\
\text { - Significant between-group difference was observed in terms of hot flashes } \\
\text { frequency ( } p=0.03) \text { and hot flashes score }(p=0.03) \text {. For both parameters, } \\
\text { differential outcome was observed, where an improvement in both } \\
\text { parameters was observed in control group, and worsening was observed } \\
\text { in intervention group. }\end{array}$ & \\
\hline
\end{tabular}


Table 4. Cont.

\begin{tabular}{|c|c|c|c|c|}
\hline \multirow[t]{6}{*}{$\begin{array}{c}\text { Menopausal } \\
\text { Symptom/Issue }\end{array}$} & Intervention Type & Intervention Name & Major Findings on Intervention Effects on Symptom * & Reference \\
\hline & & $\begin{array}{l}\text { Pharmacological intervention } \\
\text { with clonidine }\end{array}$ & $\begin{array}{l}\text { (T1: } 4 \text { weeks after randomization; T2: } 8 \text { weeks after randomization; T3: } 12 \\
\text { weeks after randomization) }\end{array}$ & $\begin{array}{l}\text { Pandya et al., } \\
2000[28]\end{array}$ \\
\hline & & & $\begin{array}{l}\text { - Intervention participants exhibited a significantly greater reduction in the } \\
\text { number of daily hot flashes }(p \leq 0.006) \text { and hot flashes scores }(p \leq 0.006) \\
\text { at T1 and T2, compared to controls. However, difference in the extent of } \\
\text { reduction between the two groups was not significant for both } \\
\text { parameters at T3. } \\
\text { - Intervention participants had a significantly greater reduction in hot } \\
\text { flashes duration only at T3 }(p=0.023) \text {, but not at T1 ( } p=0.11) \text { or T2 } \\
(p=0.18) \text {. } \\
\text { - No significant difference in extent of reduction in hot flashes severity } \\
\text { between groups at all time points of outcome assessment }(p \geq 0.08) \text {. }\end{array}$ & \\
\hline & & $\begin{array}{l}\text { Pharmacological intervention } \\
\text { with transdermal clonidine }\end{array}$ & $\begin{array}{l}\text { Intervention participants exhibited a significantly greater extent of } \\
\text { reduction in hot flashes frequency }(20 \% \text { more than controls; } p<0.0001) \text {, } \\
\text { hot flashes severity }(10 \% \text { more than controls; } p=0.02) \text { and hot flashes } \\
\text { score }(27 \% \text { more than controls; } p=0.0006) \text { as a result of the use of } \\
\text { transdermal clonidine. }\end{array}$ & $\begin{array}{l}\text { Goldberg et al., } \\
1994[29]\end{array}$ \\
\hline & $\begin{array}{l}\text { Psychotherapeutic } \\
\text { intervention }\end{array}$ & Cognitive behavioral therapy & (T1: 9 weeks after randomization; T2: 26 weeks after randomization) & $\begin{array}{l}\text { Mann et al., } \\
2012[30]\end{array}$ \\
\hline & & & $\begin{array}{l}\text { - The intervention participants exhibited a significantly greater decrease in } \\
\text { the level of perceived burden of hot flashes compared to controls, at both } \\
\text { T1 and T2 }(p<0.0001) \text {. } \\
\text { - No significant between-group differences were observed in the level of } \\
\text { reduction in hot flashes frequency, at both T1 and T2. }\end{array}$ & \\
\hline \multirow[t]{2}{*}{ Night sweats } & $\begin{array}{l}\text { Psychotherapeutic } \\
\text { intervention }\end{array}$ & Cognitive behavioral therapy & (T1: 9 weeks after randomization; T2: 26 weeks after randomization) & $\begin{array}{l}\text { Mann et al., } \\
2012[30]\end{array}$ \\
\hline & & & $\begin{array}{l}\text { - The intervention participants exhibited a significantly greater decrease in } \\
\text { the level of perceived burden of night sweats compared to controls, at } \\
\text { both T1 and T2 }(p<0.0001) \text {. } \\
\text { No significant between-group differences were observed in the level of } \\
\text { reduction in night sweats frequency, at both T1 and T2. }\end{array}$ & \\
\hline
\end{tabular}


Table 4. Cont.

\begin{tabular}{|c|c|c|c|c|}
\hline $\begin{array}{l}\text { Menopausal } \\
\text { Symptom/Issue }\end{array}$ & Intervention Type & Intervention Name & Major Findings on Intervention Effects on Symptom* & Reference \\
\hline \multirow[t]{4}{*}{ Vaginal atrophy } & \multirow[t]{4}{*}{$\begin{array}{l}\text { Miscellaneous } \\
\text { intervention }\end{array}$} & \multirow[t]{4}{*}{$\begin{array}{l}\text { Vitamin } \mathrm{D} / \text { vitamin } \mathrm{E} \text { vaginal } \\
\text { suppository intervention }\end{array}$} & $\begin{array}{l}\text { (T1: } 2 \text { weeks after start of intervention; T2: } 4 \text { weeks after start of intervention; } \\
\text { T3: } 8 \text { weeks after start of intervention) } \\
\text { Within-group comparison }\end{array}$ & \multirow[t]{4}{*}{$\begin{array}{l}\text { Keshavarzi et al } \\
2019[31]\end{array}$} \\
\hline & & & $\begin{array}{l}\text { - Significant decreases in the mean score of the genitourinary atrophy } \\
\text { self-assessment were observed among the participants in the vitamin D } \\
\text { and vitamin E groups over the 8-week intervention }(p<0.001) \text {. } \\
\text { No significant difference was observed in this score among the } \\
\text { participants in the placebo group }(p=0.564) \text {. }\end{array}$ & \\
\hline & & & Between-group comparison & \\
\hline & & & $\begin{array}{l}\text { The mean score of the genitourinary atrophy self-assessment was } \\
\text { significantly lower among the participants in the vitamin D and vitamin } \\
\text { E groups compared to controls, at T1 }(p=0.017), \mathrm{T} 2(p<0.001) \text { and T3 } \\
(p<0.001) \text {. }\end{array}$ & \\
\hline \multirow[t]{4}{*}{ Sexual issues } & $\begin{array}{l}\text { Pharmacological } \\
\text { intervention }\end{array}$ & $\begin{array}{l}\text { Pharmacological intervention } \\
\text { with tablets of a medicinal plant } \\
\text { extract (Paullinia cupana or } \\
\text { Guarana) }\end{array}$ & - $\quad$ No significant difference was observed between groups $(p=0.60)$. & $\begin{array}{l}\text { de Sousa } \\
\text { Vieira et al., } \\
2019[25]\end{array}$ \\
\hline & $\begin{array}{l}\text { Physical activity } \\
\text { intervention }\end{array}$ & $\begin{array}{l}\text { Supervised combined exercise } \\
\text { training intervention }\end{array}$ & $\begin{array}{l}\text { - No significant time } \times \text { group interaction for sexual functioning }(p=0.77) \\
\text { or sexual enjoyment }(p=0.16) \text { scores. }\end{array}$ & $\begin{array}{l}\text { Paulo et al., } \\
2019[32]\end{array}$ \\
\hline & \multirow[t]{2}{*}{$\begin{array}{l}\text { Multimodal } \\
\text { intervention }\end{array}$} & \multirow{2}{*}{$\begin{array}{l}\text { A multimodal intervention } \\
\text { involving sexual counseling, } \\
\text { and use of vaginal moisturizers, } \\
\text { lubricants, and/or dilator }\end{array}$} & (T1: 6 months after randomization; T2: 12 months after randomization) & \multirow[t]{2}{*}{$\begin{array}{l}\text { Advani et al., } \\
2017[33]\end{array}$} \\
\hline & & & $\begin{array}{l}\text { - No significant differences in \% of women with sexual dysfunction } \\
\text { between treatment groups at baseline, T1 and T2 any of the three time } \\
\text { points of data collection. } \\
\text { At T1, Active Group-H exhibited more significant improvement in sexual } \\
\text { function compared with Active Group-P, in terms of the FSFI total score } \\
(p=0.04) \text {. } \\
\text { At T1, the control participants had more dyspareunia than the } \\
\text { intervention participants did }(p=0.07) \text {. }\end{array}$ & \\
\hline
\end{tabular}

${ }^{*}$ Major findings are reported in the form of between-group comparisons unless otherwise state. 


\subsubsection{Joint Symptoms}

Ten studies reported the effect of their respective interventions on addressing joint symptoms, including joint pain and joint stiffness, among breast cancer survivors undergoing adjuvant endocrine therapy (Table 5).

Henry et al. [34] was the only group to investigate the effect of pharmacological interventions on joint symptoms. The study demonstrated that an intervention involving the intake of duloxetine, a known anti-depressant, could alleviate joint pain and joint stiffness and reduce pain interference score, where significant between-group differences were observed in these outcomes throughout the intervention. However, the intervention lacks a long-term effect in the alleviation of joint pain and stiffness, with no significant between-group differences in the aforementioned outcomes observed at 12 weeks after intervention discontinuation.

Four studies reported the effect of physical activity interventions on addressing joint symptoms. Three of the studies, which report a home-based walking program and physical activity behavior change intervention that lasted 6-12 weeks, demonstrated no significant effect on alleviating joint pain [35-37], although one reported a trend for a decrease in joint pain among the intervention participants [35]. Two reported no significant between-group differences in physical dysfunction of joints $(p \geq 0.09)$ [36,37]. In contrast, an exercise intervention lasting for one year was effective in alleviating joint pain, with significant between-group differences observed throughout the intervention for worst joint pain scores and joint pain severity [38]. Inconsistency is observed between all studies regarding the effect of exercise interventions on perceived joint stiffness, where only one study reported differential between-group outcomes on the Western Ontario and McMaster Universities Osteoarthritis Index (WOMAC) score [38]. Overall, physical activity interventions do not appear to be effective in alleviating joint pain or dysfunction among breast cancer patients undergoing adjuvant endocrine therapy, but they are potentially useful in addressing joint stiffness. Moreover, a physical activity intervention that is longer in duration could potentially be useful in addressing both joint pain and stiffness.

One study investigated the effect of a dietary intervention involving omega-3 fatty acid intake on joint symptoms [39]. Hershman et al. demonstrated a lack of effect of this intervention on reducing overall pain, joint pain and joint stiffness of participants. The intervention did not have any effect on the level of interference on daily activities caused by the above symptoms either $(p \geq 0.12)$.

Three studies reported the effect of acupuncture on addressing joint pain and stiffness [40-42]. Conflicting data were reported by these studies. While Hershman et al. reported significant between-group differences of worst joint pain scores, joint pain severity and worst joint stiffness scores at post-intervention [40], Oh et al. observed otherwise [41]. Crew et al. [42] reported that, while significant between-group difference was observed when the above parameters were assessed using Brief Pain Inventory-Short Form (BPI-SF), such significance difference was no longer observed when Western Ontario and McMaster Universities Osteoarthritis Index (WOMAC) was used for outcome measurement. Overall, firm conclusions cannot be drawn on whether acupuncture interventions can effectively address joint pain and joint stiffness.

Baker et al. [43] examined the effectiveness of a whole-body vibration intervention on joint symptom management among breast cancer survivors. This intervention was found to have no significant effect on alleviation of fatigue, joint pain, joint stiffness and physical dysfunction of joints $(p \geq 0.06)$. Additionally, the intervention had no effect on the improvement of participants' functional ability.

Overall, while a number of studies investigated the effect of interventions on addressing joint symptoms among breast cancer survivors undergoing adjuvant endocrine therapy, the inconsistency of these findings based on intervention types results in difficulties in drawing firm conclusions on which intervention type is the most appropriate for this purpose. 
Table 5. A summary of the effects of reported interventions on various endocrine therapy-induced joint symptoms.

\begin{tabular}{|c|c|c|c|}
\hline Intervention Type & Intervention Name & Major Findings on Intervention Effects on Symptom * & Reference \\
\hline $\begin{array}{l}\text { Pharmacological } \\
\text { intervention }\end{array}$ & $\begin{array}{l}\text { Pharmacological } \\
\text { intervention with } \\
\text { Duloxetine }\end{array}$ & $\begin{array}{l}\text { (T1: } 2 \text { weeks after randomization; T2: } 6 \text { weeks after randomization; T3:12 weeks after randomization; T4: } \\
\text { 24 weeks after randomization) } \\
\text { - } \quad \text { More significant decrease ( } 0.82 \text { points more) in average pain score was observed among intervention } \\
\text { participants compared to controls ( } p=0.0002) \text {. } \\
\text { - However, by T4, no significant difference in this parameter was observed between groups ( } p=0.80) \text {. } \\
\text { - Significantly more intervention participants exhibited clinically meaningful improvement in pain at } \\
\text { T2, compared to controls ( } 68 \% \text { vs. } 49 \%, p=0.003) \text {. No significant difference between groups for this } \\
\text { parameter at other time points. } \\
\text { - There was a significant improvement of joint pain in knees and hips among intervention participants, } \\
\text { compared to controls ( } p<0.001) \text {. } \\
\text { At T1, T2 and T3, participants in intervention group showed significantly lower levels of worst joint } \\
\text { pain, pain interference and joint stiffness as measured by BPI and GRCS. No significant } \\
\text { between-group difference was observed in T4. }\end{array}$ & Henry et al., 2018 [34] \\
\hline $\begin{array}{l}\text { Physical activity } \\
\text { intervention }\end{array}$ & $\begin{array}{l}\text { Home-based walking } \\
\text { program }\end{array}$ & $\begin{array}{l}\text { (T1: immediate post-intervention; T2: } 6 \text { months post-intervention) } \\
\text { Within-group comparisons } \\
\text { Intervention group } \\
\text { - } \quad \text { At T1, intervention participants experienced a decrease in joint pain, but the decrease is not } \\
\text { statistically significant ( } p \text { value not reported). Nevertheless, participants' perceived level of joint } \\
\text { stiffness ( } p<0.05 \text { ) and perceived difficulty with daily activities ( } p<0.01) \text { decreased significantly after } \\
\text { the intervention. } \\
\text { By T2, the level of joint pain and stiffness and perceived difficulty with daily activities were still } \\
\text { lower compared to baseline, but the difference was non-significant ( } p \text { value not reported). } \\
\text { Control group } \\
\text { - At immediately post-intervention, no significant differences were observed in all the above outcomes } \\
\text { when compared to baseline. } \\
\text { Between-group comparisons } \\
\text { - At T1, intervention participants had reduced stiffness scores ( } p<0.05) \text { and less difficulty with } \\
\text { activities of daily living ( } p<0.01) \\
\text { No significant between-group difference was observed for joint pain and stiffness between T1 and T2 } \\
(p \text { value not reported). }\end{array}$ & Nyrop et al., 2017 [35] \\
\hline
\end{tabular}


Table 5. Cont

\begin{tabular}{|c|c|c|c|}
\hline Intervention Type & Intervention Name & Major Findings on Intervention Effects on Symptom * & Reference \\
\hline & $\begin{array}{l}\text { Physical activity behavior } \\
\text { change intervention (The } \\
\text { BEAT Cancer } \\
\text { Program)—pilot study }\end{array}$ & $\begin{array}{l}\text { (T1: immediate post-intervention; T2: } 3 \text { months post-intervention) } \\
\text { - Between baseline and T2, no significant between-group difference was observed for the changes in } \\
\text { level of joint pain }(p=0.32) \text {, joint stiffness }(p=0.40) \text { or physical dysfunction of joints }(p=0.09) \text {. } \\
\text { Between baseline and T1, no significant between-group difference on joint pain }(p=0.40) \text { or physical } \\
\text { dysfunction of joints }(p=0.41) \text {, but more significant improvement on joint stiffness was observed } \\
\text { among intervention participants }(p=0.04) \text {. }\end{array}$ & $\begin{array}{l}\text { Rogers et al., } 2009 \text { [36] } \\
\text { Rogers et al., } 2009 \text { [37] }\end{array}$ \\
\hline & $\begin{array}{l}\text { Year-long exercise } \\
\text { intervention }\end{array}$ & $\begin{array}{l}\text { (T1: } 3 \text { months after randomization; T2: } 6 \text { months after randomization; T3: } 9 \text { months after randomization; } \\
\text { T4: } 12 \text { months after randomization) } \\
\text { - Worst joint pain score was decreased by } 29 \% \text { among intervention participants, while increased by } 3 \% \\
\text { among control participants at T4. ( } p<0.001) \text {. } \\
\text { - Statistically significant difference was also observed in joint pain severity between intervention and } \\
\text { control participants. }(p<0.001) \text {. } \\
\text { - WOMAC total score (measure of joint symptoms in lower limbs) was decreased by } 37 \% \text { among } \\
\text { intervention participants, while increased by } 2 \% \text { among control participants at T4 }(p<0.001) .\end{array}$ & Irwin et al., 2015 [38] \\
\hline Dietary intervention & $\begin{array}{l}\text { Dietary intervention with } \\
\text { omega-3 fatty acids }\end{array}$ & $\begin{array}{l}\text { (T1: Week } 6 \text { of intervention; T2: Week } 12 \text { of intervention; T3: Week } 24 \text { of intervention/immediate } \\
\text { post-intervention) } \\
\text { Within-group comparisons } \\
\text { - There was significant reduction in pain from baseline for both intervention and control groups at T1, } \\
\text { T2 and T3 }(p<0.01) \text {. } \\
\text { Between-group comparisons } \\
\text { - There was no significant difference in the worst pain score assessed by BPI between intervention and } \\
\text { control groups at all of the time points of measurement }(p \geq 0.34) \text {. } \\
\text { - Similar observations were obtained for level of interference on daily activities by pain ( } p \geq 0.58) \text {, } \\
\text { global rating in change in joint pain ( } p \geq 0.16) \text { and joint stiffness pain }(p \geq 0.12) \text {. } \\
\text { There were no significant differences in the perceived joint pain levels (as measured by WOMAC and } \\
\text { MSACRAH) ( } p \geq 0.41) \text { and serum CRP levels (objective measure of joint pain, } p=0.71 \text { ) at all the time } \\
\text { points of measurement between intervention and control groups. }\end{array}$ & $\begin{array}{l}\text { Hershman et al., } 2015 \\
\text { [39] }\end{array}$ \\
\hline
\end{tabular}


Table 5. Cont.

\begin{tabular}{|c|c|c|c|}
\hline Intervention Type & Intervention Name & Major Findings on Intervention Effects on Symptom * & Reference \\
\hline Acupuncture intervention & Acupuncture intervention & $\begin{array}{l}\text { (T1: } 6 \text { weeks after randomization; T2: } 12 \text { weeks after randomization) } \\
\text { Worst joint pain score } \\
\text { - The worst joint pain score among the intervention participants was significantly lower than that } \\
\text { among participants in both control groups at T1 (lower by } 0.92-0.96 \text { points, } p=0.01 \text { ). } \\
\text { - However, the between-group difference in this outcome was no longer significant at T2 when } \\
\text { comparing between intervention group and sham acupuncture control group }(p=0.08 \text { ). } \\
\text { Average joint pain } \\
\text { - The average pain score among the intervention participants was significantly lower than that among } \\
\text { participants in both control groups at both T1 (lower by } 0.60-0.71 \text { points, } p \leq 0.04 \text { ) and T2 (lower by } \\
0.79-1.38 \text { points, } p \leq 0.02 \text { ). } \\
\text { Joint pain severity } \\
\text { - The pain severity score among the intervention participants was significantly lower than that among } \\
\text { participants in both control groups at T1 (lower by } 0.56-0.71 \text { points, } p \leq 0.05 \text { ). } \\
\text { - However, at T2, no significant difference in this parameter was observed between intervention group } \\
\text { and sham acupuncture control group (lower by } 0.53 \text { points, } p=0.08 \text { ). } \\
\text { Worst joint stiffness score } \\
\text { - The worst joint stiffness score among the intervention participants was significantly lower than that } \\
\text { among participants in both control groups at T1 (lower by } 1.00-1.09 \text { points, } p \leq 0.02 \text { ). } \\
\text { However, at T2, no significant difference in this parameter was observed between intervention group } \\
\text { and sham acupuncture control group (lower by } 0.72 \text { points, } p=0.08 \text { ). }\end{array}$ & $\begin{array}{l}\text { Hershman et al, } 2018 \\
\text { [40] }\end{array}$ \\
\hline & $\begin{array}{l}\text { Electro-acupuncture } \\
\text { intervention }\end{array}$ & $\begin{array}{l}\text { (T1: Immediate post-intervention; T2: } 6 \text { months post-intervention) } \\
\text { - No significant between-group differences were observed in joint pain and joint stiffness at both T1 } \\
\text { and T2 ( } p \text { values not reported). } \\
\text { - However, there was a trend of higher level of improvement in joint stiffness and physical functioning } \\
\text { at T2 for the intervention participants, compared with controls. }\end{array}$ & Oh et al., 2013 [41] \\
\hline
\end{tabular}


Table 5. Cont

\begin{tabular}{|c|c|c|c|}
\hline Intervention Type & Intervention Name & Major Findings on Intervention Effects on Symptom * & Reference \\
\hline & Acupuncture intervention & $\begin{array}{l}\text { Within-group comparisons } \\
\text { - As measured by BPI-SF for joint pain measurement, significant decrease in worst pain score } \\
(p=0.008), \text { pain severity }(p=0.022) \text { and pain-related interference }(p=0.015) \text { was reported among the } \\
\text { intervention participants after receiving acupuncture. } \\
\text { As measured by WOMAC, improvement of joint pain }(p=0.145) \text { and joint stiffness }(p=0.067) \text { was } \\
\text { observed among intervention participants after receiving acupuncture, but the level of improvement } \\
\text { did not reach statistical significance. } \\
\text { Between-group comparisons } \\
\text { - The opposite effect was observed in the changes of pain severity, joint pain and stiffness between the } \\
\text { intervention and control participants ( } p \text { values not reported). } \\
\text { Despite the improvement of symptoms among intervention participants, such improvement did not } \\
\text { persist } 6 \text { weeks after the intervention. }\end{array}$ & Crew et al., 2007 [42] \\
\hline $\begin{array}{l}\text { Miscellaneous } \\
\text { intervention }\end{array}$ & $\begin{array}{l}\text { Whole body vibration } \\
\text { intervention }\end{array}$ & $\begin{array}{l}\text { No significant differences in the joint pain levels }(p=0.334) \text {, joint stiffness levels }(p=0.224) \text { or level of } \\
\text { physical dysfunction of joints ( } p=0.063 \text { ) of participants between groups at post-intervention. }\end{array}$ & Baker et al., 2018 [43 \\
\hline
\end{tabular}

${ }^{*}$ Major findings are reported in the form of between-group comparisons unless otherwise stated. 


\subsubsection{Fatigue}

Intervention effects on fatigue among breast cancer survivors undergoing adjuvant endocrine therapy were investigated in seven studies (Table 6).

Zhao et al. [44] reported the only study that examined the effect of pharmacological interventions on fatigue levels. They found that the intake of spore powder from G. lucidum would lead to a significant improvement of fatigue $(p<0.01)$. This finding was further supported by the observation that such an intervention could significantly lower participants' serum TNF-alpha and IL-6 levels $(p<0.01)$, which are shown to have linear correlations with cancer-related fatigue.

Two studies assessed the effects of physical activity interventions on fatigue levels, and conflicting data were obtained. While a significant difference in fatigue levels $(p=0.001)$ was demonstrated between groups and over time by Paulo et al. [32] who reported an exercise program, Nyrop et al. [35] who reported a home-based walking program reported no significant between-group differences at post-intervention. It is therefore difficult to draw firm conclusions on the effect of physical activity interventions on fatigue levels.

Using a combination of instruments for fatigue assessment, Peppone et al. showed that yoga intervention involving mindfulness exercises could reduce fatigue symptoms significantly $(p=0.001)$ among the participants having received the intervention [45], demonstrating the potential of similar psychotherapeutic interventions to address this symptom.

Mao et al. conducted the sole study in this review to report the effect of acupuncture intervention on fatigue [46]. They reported that participants who received electro-acupuncture exhibited a significantly greater extent of improvement in fatigue levels at post-intervention $(p=0.0095)$, and this effect was sustained at four weeks post-intervention $(p=0.022)$.

The effect of a whole-body vibration intervention and neuromuscular taping intervention on reducing fatigue among the survivors was assessed by Baker et al. [43] and Conejo et al. [47], respectively. The whole-body vibration intervention exhibited no significant effect on improving fatigue at post-intervention $(p=0.079)$. The neuromuscular taping intervention appeared to have significantly alleviated fatigue, albeit a need for a longer duration post-intervention to take effect. Significant between-group differences in fatigue severity was only observed at five weeks after receiving this intervention. 
Table 6. A summary of the effects of reported interventions on endocrine therapy-induced fatigue.

\begin{tabular}{|c|c|c|c|}
\hline Intervention Type & Intervention Name & Major Findings on Intervention Effects on Fatigue * & Reference \\
\hline $\begin{array}{l}\text { Pharmacological } \\
\text { intervention }\end{array}$ & $\begin{array}{l}\text { Pharmacological } \\
\text { intervention with spore } \\
\text { powder of G. lucidum }\end{array}$ & $\begin{array}{l}\text { - Compared to control participants, intervention participants had a more significant } \\
\text { improvement FACT-F score }(p<0.01) \text {. }\end{array}$ & Zhao et al., 2012 [44] \\
\hline \multirow[t]{3}{*}{$\begin{array}{l}\text { Physical activity } \\
\text { intervention }\end{array}$} & $\begin{array}{l}\text { Supervised combined } \\
\text { exercise training } \\
\text { intervention }\end{array}$ & $\begin{array}{l}\text { - There was a significant time } \times \text { group interaction for the perceived severity of } \\
\text { fatigue among the intervention participants, compared to controls. }(p=0.001) \text {. }\end{array}$ & Paulo et al., 2019 [32] \\
\hline & $\begin{array}{l}\text { Home-based walking } \\
\text { program }\end{array}$ & $\begin{array}{l}\text { (T1: immediate post-intervention; T2: } 6 \text { months post-intervention) } \\
\text { Within-group comparisons } \\
\text { - Both groups exhibited no significant changes on fatigue level as measured by VAS } \\
\text { at T1, when compared to baseline. }\end{array}$ & Nyrop et al., 2017 [35] \\
\hline & & $\begin{array}{l}\text { Between-group comparisons } \\
\text { - There was no significant between-group difference on fatigue level between T1 } \\
\text { and T2 ( } P \text { values not reported). }\end{array}$ & \\
\hline $\begin{array}{l}\text { Psychotherapeutic } \\
\text { intervention }\end{array}$ & Yoga intervention & $\begin{array}{l}\text { - Intervention participants also perceived a significantly greater reduction in fatigue } \\
\text { levels indicated by the FACIT-F physical subscale score and MFSI-SF physical } \\
\text { subscale score at post-intervention (both } p=0.001 \text { ). }\end{array}$ & $\begin{array}{l}\text { Peppone et al., } 2015 \\
{[45]}\end{array}$ \\
\hline Acupuncture intervention & $\begin{array}{l}\text { Electro-acupuncture } \\
\text { intervention }\end{array}$ & $\begin{array}{l}\text { (T1: Week } 2 \text { of intervention; T2: Week } 4 \text { of intervention; T3: Week } 8 \text { of } \\
\text { intervention/immediate post-intervention; T4: } 4 \text { weeks post-intervention) } \\
\text { - } \quad \text { Intervention participants showed more significant improvement in fatigue over } \\
\text { time compared with wait-list control participants ( } p=0.0095) \text {. } \\
\text { - Greater reduction in BFI score (measure of fatigue) was observed among } \\
\text { intervention participants compared to wait-list controls at T3 }(p=0.0034) \text {, and this } \\
\text { reduction effect persisted at T4 }(p=0.022) \text {. }\end{array}$ & Mao et al., 2014 [46] \\
\hline
\end{tabular}


Table 6. Cont.

\begin{tabular}{|c|c|c|c|}
\hline Intervention Type & Intervention Name & Major Findings on Intervention Effects on Fatigue * & Reference \\
\hline \multirow[t]{7}{*}{$\begin{array}{l}\text { Miscellaneous } \\
\text { intervention }\end{array}$} & $\begin{array}{l}\text { Whole body vibration } \\
\text { intervention }\end{array}$ & $\begin{array}{l}\text { - There were no significant differences in the perceived fatigue levels }(p=0.079) \text { of } \\
\text { participants between groups at post-intervention. }\end{array}$ & Baker et al., 2018 [43] \\
\hline & $\begin{array}{l}\text { Neuromuscular taping } \\
\text { intervention }\end{array}$ & $\begin{array}{l}\text { (T1: } 1 \text { week after start of intervention/immediate post-intervention; T2: } 5 \text { weeks after } \\
\text { start of intervention/4 weeks post-intervention) } \\
\text { Within-group comparisons } \\
\text { Intervention group }\end{array}$ & Conejo et al., 2018 [47] \\
\hline & & $\begin{array}{l}\text { - Significant improvement was observed for fatigue }(p=0.00) \text { between baseline, T1 } \\
\text { and T2. }\end{array}$ & \\
\hline & & Control group & \\
\hline & & $\begin{array}{l}\text { - No significant improvement was observed for fatigue }(p \geq 0.46) \text { between baseline, } \\
\text { T1 and T2. }\end{array}$ & \\
\hline & & Between-group comparisons & \\
\hline & & $\begin{array}{l}\text { - There was no significant between-group difference in fatigue score at } \mathrm{T} 1(p=0.07) \\
\text { - However, by T2, intervention group had significantly higher score for fatigue } \\
(p=0.01) \text { compared to controls. }\end{array}$ & \\
\hline
\end{tabular}

${ }^{*}$ Major findings are reported in the form of between-group comparisons unless otherwise stated. 


\subsubsection{Sleep Disturbance}

Seven studies involved sleep disturbance as an outcome for assessing the effectiveness of interventions for breast cancer survivors undergoing adjuvant endocrine therapy (Table 7).

Four studies examined the effect of physical activity interventions on addressing sleep disturbance and/or improving sleep quality $[32,36,37,48]$. While two of the studies reported a significantly greater decrease in sleep disturbance $(p \leq 0.04)$ and improvement in sleep quality $(p=0.002)$ immediately post-intervention [32,48], the two other studies reported a lack of significant difference on these parameters [36,37]. Notably, the physical activity behavior change intervention reported by Rogers et al. [48] no longer exhibited a beneficial effect on alleviating sleep disturbance and improving sleep quality at three-month post-intervention, suggesting that the intervention may not exhibit long-term effectiveness, and it prompts a need for continuous practice of the intervention for it to take effect.

Cognitive behavioral therapy, a type of psychotherapeutic interventions, was shown by Mann et al. [30] to significantly improve participants' sleep quality at post-intervention $(p<0.001)$, and this beneficial effect persisted for a further 17 weeks $(p<0.05)$. These data suggest the potential of this therapy to be implemented for addressing sleep problems among breast cancer survivors.

In contrast, acupuncture intervention may not be as effective in addressing sleep problems. In a study involving an electro-acupuncture intervention reported by Mao et al. [46], the authors reported a lack of significant difference in the improvement of the Pittsburgh Sleep Quality Index (PSQI) score between groups $(p \geq 0.058)$ at four- and eight-week follow-up. These data show that such intervention is unlikely to be appropriate for improving sleep quality among breast cancer survivors in both the short and long term.

Likewise, the neuromuscular taping intervention appeared ineffective in addressing sleep problems, as evidenced by the lack of significant between-group difference in the perceived levels of insomnia among the participants at both immediate post-intervention and four weeks post-intervention [47]. 
Table 7. A summary of the effects of reported interventions on endocrine therapy-induced sleep disturbance.

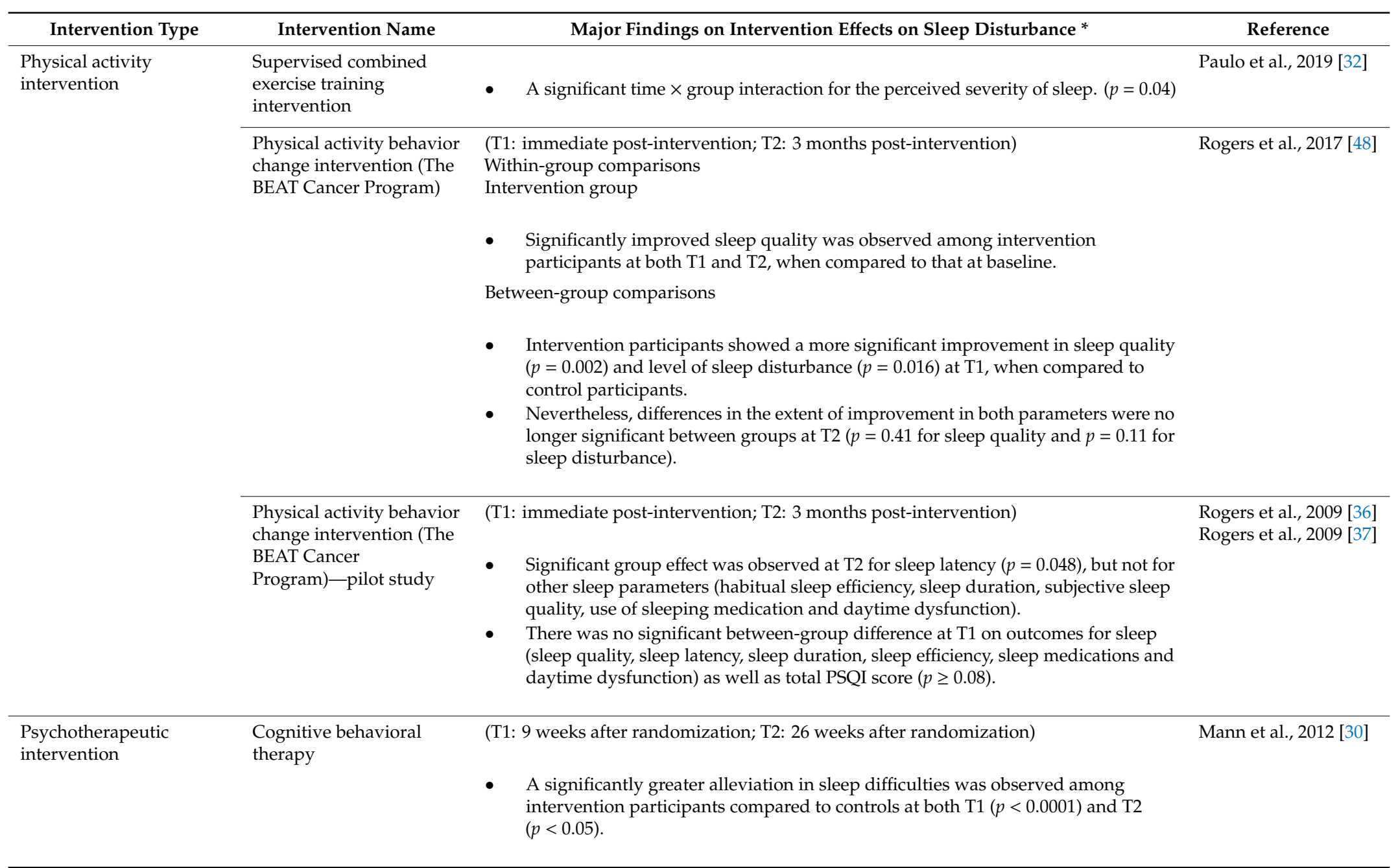


Table 7. Cont.

\begin{tabular}{|c|c|c|c|}
\hline Intervention Type & Intervention Name & Major Findings on Intervention Effects on Sleep Disturbance * & Reference \\
\hline Acupuncture intervention & $\begin{array}{l}\text { Electro-acupuncture } \\
\text { intervention }\end{array}$ & $\begin{array}{l}\text { (T1: Week } 2 \text { of intervention; T2: Week } 4 \text { of intervention; T3: Week } 8 \text { of } \\
\text { intervention/immediate post-intervention; T4: } 4 \text { weeks post-intervention) } \\
\text { - There were no significant differences in the extent of improvement over time on } \\
\text { PSQI score (measure of sleep quality) between intervention and wait-list control } \\
\text { group }(p=0.058) \text {. } \\
\text { No significant improvement was observed among intervention participants in the } \\
\text { PSQI score at T3 }(p=0.087) \text {. }\end{array}$ & Mao et al., 2014 [46] \\
\hline $\begin{array}{l}\text { Miscellaneous } \\
\text { intervention }\end{array}$ & $\begin{array}{l}\text { Neuromuscular taping } \\
\text { intervention }\end{array}$ & $\begin{array}{l}\text { (T1: } 1 \text { week after start of intervention/immediate post-intervention; T2: } 5 \text { weeks after } \\
\text { start of intervention/4 weeks post-intervention) } \\
\text { Within-group comparisons } \\
\text { Intervention group } \\
\text { - Significant improvement was observed for insomnia }(p \leq 0.009) \text { between baseline, } \\
\text { T1 and T2. } \\
\text { Control group } \\
\text { - Significant improvement was also observed for insomnia }(p \leq 0.02) \text { between } \\
\text { baseline, } \mathrm{T} 1 \text { and T2. } \\
\text { Between-group comparisons } \\
\text { - No significant between-group differences were observed }(p=1.00) \text { for insomnia at } \\
\text { T1 and T2. }\end{array}$ & Conejo et al., 2018 [47] \\
\hline
\end{tabular}

${ }^{*}$ Major findings are reported in the form of between-group comparisons unless otherwise stated. 


\subsubsection{QOL/HRQOL}

Thirteen studies reported the effects of their respective intervention on QOL or HRQOL outcomes of breast cancer survivors undergoing adjuvant endocrine therapy, and two studies reported that on functional ability of these survivors (Table 8).

There were five studies reporting different pharmacological interventions on survivors' QOL. Three of the studies demonstrated that such interventions could lead to an improvement on this outcome. Zhao et al. [44] reported a significantly greater extent of improvement in multiple domains of QOL among participants who took spore powder of G. lucidum compared to control. Functional QOL was also reported by Henry et al. to have been more significantly improved among participants who took duloxetine [34]. An intervention involving clonidine intake could also lead to a short-term improvement on patients' QOL $(p \leq 0.022)$ at four- and eight-week follow-up, but this effect was no longer observed at Week $12(p>0.20)$ [28]. In contrast, pharmacological interventions involving the use of sertraline or Guarana appeared to have no effect on patients' QOL $[25,27]$.

Four studies reported the effect of physical activity interventions on QOL outcomes. Participants who received the supervised combined exercise training intervention were reported to exhibit a greater extent of improvement on this outcome [32], suggesting the effectiveness of an intervention combining resistance, aerobic and stretching exercises in QOL improvement. Rogers et al. [36,37] also showed in a pilot study that the physical activity behavior change intervention comprising supervised exercise, home-based exercise and physical activity counselling sessions exhibited significant effects in improving social well-being and overall QOL, although such effect was only exhibited at three months post-intervention. In contrast, a home-based walking program appeared to have no effect in QOL improvement, as shown by the insignificant differences of FACT-G scores at post-intervention between participants who received the program and the control counterparts [35].

Cognitive behavioral therapy was also shown to have some beneficial effect on survivors' HRQOL [30]. Participants who received this therapy were reported to have a significantly greater extent of improvement on general health, physical functioning and social functioning than controls, although significant improvement for the latter two outcomes was only observed later at Week 26 of the study.

Conflicting findings were obtained regarding the effect of acupuncture interventions on survivors' QOL. While implementation of an acupuncture intervention would enable participants to exhibit a significantly greater extent of QOL improvement [42], that of an electro-acupuncture intervention failed to do so [41]. Note that, while the significant effect of QOL improvement exhibited by the acupuncture intervention did not persist in the long term [42], there was a trend for increased improvement of physical functioning among participants who received the electro-acupuncture intervention at a longer-term follow-up [41].

Finally, the neuromuscular taping intervention was found to be effective in the improvement in participants' perceived global health status and QOL [47]. A more significant improvement in these parameters was only observed among the intervention participants at five weeks after receiving the intervention $(p=0.005)$ and not immediately post-intervention. This suggests a need for a longer duration for the intervention to take effect. 
Table 8. A summary of the effects of reported interventions on participants' QOL and functional ability.

\begin{tabular}{|c|c|c|c|c|}
\hline Outcome & Intervention Type & Intervention Name & $\begin{array}{l}\text { Major Findings on Intervention Effects on QOL/Functional } \\
\text { Ability * }\end{array}$ & Reference \\
\hline \multirow[t]{5}{*}{ QOL } & $\begin{array}{l}\text { Pharmacological } \\
\text { intervention }\end{array}$ & $\begin{array}{l}\text { Pharmacological intervention } \\
\text { with tablets of a medicinal plant } \\
\text { extract (Paullinia cupana or } \\
\text { Guarana) }\end{array}$ & $\begin{array}{l}\text { - No significant difference was observed between groups on } \\
\text { participants' QOL ( } p \text { value not reported). }\end{array}$ & de Sousa Vieira et al., 2019 [25] \\
\hline & & $\begin{array}{l}\text { Pharmacological intervention } \\
\text { with Duloxetine }\end{array}$ & $\begin{array}{l}\text { - There was significant improvement of functional QOL among } \\
\text { intervention participants, compared to controls }(p=0.009) \text {. }\end{array}$ & Henry et al., 2018 [34] \\
\hline & & $\begin{array}{l}\text { Pharmacological intervention } \\
\text { with spore powder of G. lucidum }\end{array}$ & $\begin{array}{l}\text { There was a more significant improvement in scores of various } \\
\text { QOL domains, including emotional functioning }(p<0.05) \text {, } \\
\text { cognitive functioning }(p<0.05) \text {, physical functioning }(p<0.01) \\
\text { and global QOL }(p<0.01) \text {. }\end{array}$ & Zhao et al., 2012 [44] \\
\hline & & $\begin{array}{l}\text { Pharmacological intervention } \\
\text { with the antidepressant } \\
\text { sertraline }\end{array}$ & $\begin{array}{l}\text { (T1: Before cross-over at } 6 \text { weeks after start of intervention; T2: After } \\
\text { cross-over at } 12 \text { weeks after start of intervention) } \\
\text { - No significant difference was observed in FACT-B score between } \\
\text { groups at both before cross-over at T1 }(p=0.32) \text { and after } \\
\text { cross-over at T2 }(p=0.88) \text {. }\end{array}$ & Kimmick et al., 2006 [27] \\
\hline & & $\begin{array}{l}\text { Pharmacological intervention } \\
\text { with clonidine }\end{array}$ & $\begin{array}{l}\text { (T1: } 4 \text { weeks after randomization; T2: } 8 \text { weeks after randomization; } \\
\text { T3: } 12 \text { weeks after randomization) } \\
\text { - Compared to controls, intervention participants exhibited a } \\
\text { significantly greater improvement QOL score at T1 }(p=0.003) \\
\text { and T2 ( } p=0.022) \text {, but the difference in the extent of such } \\
\text { improvement between groups was not significant at T3 ( } p> \\
\text { 0.20). }\end{array}$ & Pandya et al., 2000 [28] \\
\hline
\end{tabular}


Table 8. Cont.

\begin{tabular}{|c|c|c|c|c|}
\hline Outcome & Intervention Type & Intervention Name & $\begin{array}{c}\text { Major Findings on Intervention Effects on QOL/Functional } \\
\text { Ability * }\end{array}$ & Reference \\
\hline & \multirow[t]{8}{*}{$\begin{array}{l}\text { Physical activity } \\
\text { intervention }\end{array}$} & $\begin{array}{l}\text { Supervised combined exercise } \\
\text { training intervention }\end{array}$ & $\begin{array}{l}\text { (T1: } 12 \text { weeks after start of intervention; T2: } 24 \text { weeks after start of } \\
\text { intervention; T3: } 36 \text { weeks after start of intervention) }\end{array}$ & Paulo et al., 2019 [32] \\
\hline & & & $\begin{array}{l}\text { A significant time } \times \text { group interaction was observed for the } \\
\text { scores for role functioning domain in EORTC-CLC-C } 30 \\
(p=0.01) \text {, and most of the domains in SF-36 }(p \leq 0.02) \text { at T2 } \\
\text { and T3. }\end{array}$ & \\
\hline & & \multirow[t]{6}{*}{ Home-based walking program } & $\begin{array}{l}\text { (T1: immediate post-intervention; T2: } 6 \text { weeks post-intervention) } \\
\text { Within-group comparisons } \\
\text { Intervention group }\end{array}$ & \multirow[t]{6}{*}{ Nyrop et al., 2017 [35] } \\
\hline & & & $\begin{array}{l}\text { - No significant changes were observed among intervention } \\
\text { participants the score for emotional well-being and functional } \\
\text { well-being (as measured by FACT-G) at T1, when compared } \\
\text { to baseline. }\end{array}$ & \\
\hline & & & Control group & \\
\hline & & & $\begin{array}{l}\text { - At T1, none of the FACT-G domains exhibited significant } \\
\text { differences when compared to baseline. }\end{array}$ & \\
\hline & & & Between-group comparisons & \\
\hline & & & $\begin{array}{l}\text { - There was no significant between-group difference on the scores } \\
\text { of all FACT-G domains between T1 and T2. }\end{array}$ & \\
\hline & & $\begin{array}{l}\text { Physical activity behavior } \\
\text { change intervention (The BEAT } \\
\text { Cancer Program)—pilot study }\end{array}$ & $\begin{array}{l}\text { (T1: immediate post-intervention; T2: } 3 \text { months post-intervention) } \\
\text { - } \quad \text { Between baseline and T1, no significant between-group } \\
\text { differences were observed in overall QOL or any sub-scales of } \\
\text { FACT-B, except a significantly greater extent of improvement on } \\
\text { social well-being }(p=0.03) \text {. } \\
\text { Between baseline and T2, a significantly greater extent of } \\
\text { improvement on social well-being }(p=0.03) \text { and overall QOL } \\
(p=0.045) \text { was noted for intervention group. However, none of } \\
\text { the other subscales of FACT-B (physical well-being, emotional } \\
\text { well-being, functional well-being and additional concerns) } \\
\text { showed significant between-group differences. }\end{array}$ & $\begin{array}{l}\text { Rogers et al., } 2009[36] \\
\text { Rogers et al., } 2009[37]\end{array}$ \\
\hline
\end{tabular}


Table 8. Cont

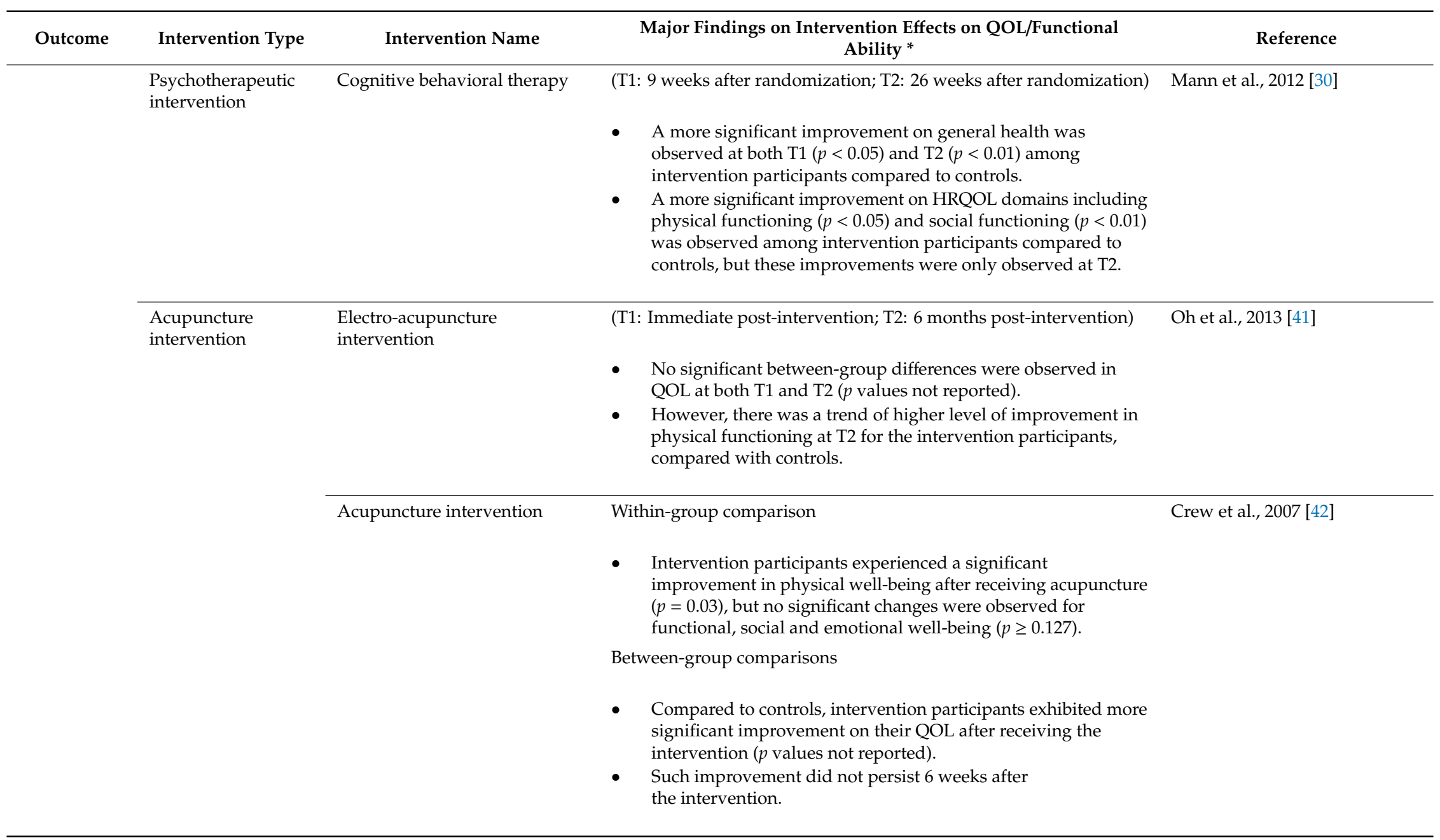


Table 8. Cont.

\begin{tabular}{|c|c|c|c|c|}
\hline Outcome & Intervention Type & Intervention Name & $\begin{array}{l}\text { Major Findings on Intervention Effects on QOL/Functional } \\
\text { Ability * }\end{array}$ & Reference \\
\hline & $\begin{array}{l}\text { Miscellaneous } \\
\text { intervention }\end{array}$ & $\begin{array}{l}\text { Neuromuscular taping } \\
\text { intervention }\end{array}$ & $\begin{array}{l}\text { (T1: } 1 \text { week after start of intervention/immediate post-intervention; } \\
\text { T2: } 5 \text { weeks after start of intervention/4 weeks post-intervention) } \\
\text { Within-group comparisons } \\
\text { Intervention group }\end{array}$ & Conejo et al., 2018 [47] \\
\hline & & & $\begin{array}{l}\text { - Significant improvement was observed in global health status } \\
(p=0.002) \text {, role functioning }(p=0.03) \text { and emotional functioning } \\
(p=0.008) \text { between baseline and T1. } \\
\text { By T2, significant improvement was observed among all } \\
\text { functional scales in the EORTC-CLC-C30 }(p<0.02) \text { and global } \\
\text { health status }(p=0.000) \text {. }\end{array}$ & \\
\hline & & & $\begin{array}{l}\text { Control group } \\
\text { No significant improvement was observed for all QOL domains at T1 } \\
\text { and T2 }(p \geq 0.41) \text {. }\end{array}$ & \\
\hline & & & Between-group comparisons & \\
\hline & & & $\begin{array}{l}\text { - No significant differences were observed in all QOL outcomes } \\
\text { between the intervention and control groups at T1 }(p \geq 0.06) \text {. } \\
\text { However, by T2, intervention group had significantly higher } \\
\text { score for global health status/QOL ( } p=0.005) \text {, compared } \\
\text { to controls. }\end{array}$ & \\
\hline \multirow[t]{2}{*}{$\begin{array}{l}\text { Functional } \\
\text { ability }\end{array}$} & $\begin{array}{l}\text { Physical activity } \\
\text { intervention }\end{array}$ & Home-based walking program & $\begin{array}{l}\text { A more significant improvement in the level of difficulties with } \\
\text { activities of daily living owing to the joint symptoms was } \\
\text { observed among intervention participants, compared to controls } \\
(p<0.01) \text {. }\end{array}$ & Nyrop et al., 2017 [35] \\
\hline & $\begin{array}{l}\text { Miscellaneous } \\
\text { intervention }\end{array}$ & $\begin{array}{l}\text { Whole body vibration } \\
\text { intervention }\end{array}$ & $\begin{array}{l}\text { - No significant differences in the changes in functional ability } \\
\text { between participants in both groups, as indicated in the results } \\
\text { for chair rise }(p=0.292) \text { and stair climb }(p=0.154) \text { exercises. }\end{array}$ & Baker et al., 2018 [43] \\
\hline
\end{tabular}

${ }^{*}$ Major findings are reported in the form of between-group comparisons unless otherwise stated. 


\subsubsection{Functional Ability}

Two studies, reporting a home-based walking program and a whole body vibration intervention, examined the effect of their respective intervention on functional ability of breast cancer survivors undergoing adjuvant endocrine therapy (Table 8). While intervention participants who received the home-based walking program experienced a significantly greater reduction in their difficulty with activities involved in their daily living [35], those who received the whole body vibration intervention reported no significant improvement in their functional ability, as measured by their ability to rise from a chair and climbing stairs [43].

\section{Discussion}

Overall, pharmacological interventions were reported to result in a perceived improvement in patients' QOL, suggesting a potential for using pharmacological products in enhancing patients' well-being. We also noted that these interventions appear to have different effects on symptoms such as hot flashes. This is not surprising because these reported interventions utilized different pharmacological products. For example, clonidine and potentially sertraline were shown to minimize hot flashes in earlier studies [27-29]. However, homeopathic medicine and Guarana extracts were reported to have no effect on alleviation of this symptom $[25,26]$. Indeed, one might also argue that the methodological quality of these studies could contribute to these conflicting findings. However, studies showing a positive effect of their interventions on hot flashes appeared to have mixed ratings. Among the three studies [27-29] that reported such positive effect, two were rated weak and one has a strong global rating. Meanwhile, another study [26] did not report any effect of the intervention on hot flashes, despite its strong global rating of its methodological quality. Thus, differences in quality ratings of the studies are unlikely to be a factor that can account for the discrepancies of findings.

Interestingly, clonidine, a pharmacological product for treatment of hypertension, can also exhibit alleviating effect on hot flashes. This finding prompts that pharmacological products intended for use to treat a certain non-adjuvant endocrine therapy-induced symptom could also have beneficial effects on addressing common symptoms induced by this therapy. Such findings suggest a value for conducting further studies to explore the beneficial effect of widely-used cancer palliative medications known to have minimal side effects in addressing various symptoms [49]. This would help establish the most suitable medication plan in pharmacological interventions for supportive care among patients with optimal effectiveness.

In addition, intake of traditional Chinese medicine such as the spore powder of G. lucidum would have beneficial effects in the management of cancer-associated symptoms including fatigue and improving QOL [44]. It is likely that the bioactive compounds present in G. lucidum, such as triterpenoids [50], could play a role in mediating these effects. Indeed, triterpenoids were shown to exhibit anti-oxidative effects [51], which are known to be beneficial in fatigue reduction [52]. Moreover, triterpenoids would also play a role in fatigue reduction through modulation of cytokine expression [52]. The findings by Zhao et al. [44] may therefore make a case for the exploration of dietary or medicinal products containing anti-oxidative and immunomodulatory bioactive compounds that can be used in interventions for addressing cancer-associated symptoms.

Despite the potential of pharmacological interventions in symptom management among breast cancer survivors, these interventions were also reported to induce undesirable side effects. For example, although clonidine was shown to be useful for addressing hot flashes among patients, it was also reported to contribute to symptoms including drowsiness, sleep difficulties, dry mouth and constipation [28,29]. Likewise, despite its effectiveness in treating fatigue, pharmacological intervention involving the intake of G. lucidum could also cause mild symptoms such as dry mouth and dizziness [44]. In general, side effects of drugs used should be taken into account when pharmacological interventions are designed to manage particular symptoms.

Our review also demonstrates that certain non-pharmacological interventions, which were suggested to be less prone to the occurrence of undesirable side effects [53], can be effective in symptom 
management and enhancing QOL. For example, psychotherapeutic interventions such as yoga could effectively reduce fatigue among breast cancer survivors, while cognitive behavioral therapy could help address sleep disturbance, enhance survivors' QOL, and reduce their perceived burden of hot flashes and night sweats. Acupuncture and electro-acupuncture interventions can likewise address fatigue and potentially joint pain and stiffness. Although conflicting findings were noted between studies reporting the effect of acupuncture interventions on joint symptoms $[40,41]$, we believe that such discrepancies could be contributed by the different sample size used in these studies. While the study with 226 participants indicated a significant effect of an acupuncture intervention on reducing joint symptoms [40], the study with only 29 participants reported otherwise [41]. Owing to the effect of sample sizes on the significance of between-group differences of clinical effects, future studies should consider the use of larger sample sizes for the examination of the effects of interventions on patient outcomes.

Conflicting data were obtained on the effect of physical activity interventions on all of the assessed outcomes. In our review, the included studies reported physical activity interventions involving different types of exercises including aerobic exercises, walking, use of cycle ergometers and home-based exercises. One may perceive that the intensity of exercise involved in the intervention could be a factor for the discrepancies observed, as evidenced by the difference in the effect of a home-based walking program and a program combining resistance and aerobic exercises on joint pain $[35,38]$. However, as reported in a recent meta-analysis, interventions involving different types of exercises were consistently shown to reduce pain, fatigue and insomnia among patients of various cancer types [54]. This finding prompts that intensity of exercise involved in an intervention is unlikely to have affected intervention effectiveness. One may also argue that participants' adherence to the physical activity interventions could affect patient outcomes. Indeed, Paulo et al. [32] and Irwin et al. [38] both reported good participant adherence to the intervention, and that their intervention could lead to significant improvement of outcomes including sleep, fatigue, joint pain and QOL. However, as demonstrated by Rogers et al. [37], even though participants' adherence was reported to be over 95\%, patient outcomes such as joint pain were not significantly improved. It is therefore unlikely that participants' adherence to the intervention could play a significant role in modifying intervention effectiveness. We therefore hypothesize that the aforementioned discrepancies of findings are due to the duration of the reported interventions. For example, while shorter physical activity interventions were found not to be effective in addressing joint pain, a year-long physical activity intervention reported by Irwin et al. [38] appeared to be effective in doing so. Likewise, while longer interventions lasting nine months were found to have significant effect on the reduction of patients' fatigue [32], the six-week program reported by Nyrop et al. appeared to have no effect [35]. These data suggest that implementation of interventions that last longer could be more effective in symptom management, and future studies should take intervention duration into account to enhance intervention effectiveness.

It is also worth noting that non-pharmacological interventions that target specific symptoms were shown to be particularly effective in symptom management and improving patients' well-being. For example, an intervention that specifically targets vaginal symptoms, such as vitamin $\mathrm{D} / \mathrm{E}$ vaginal suppository intervention, can effectively alleviate vaginal atrophy, a common menopausal symptom among breast cancer patients [31]. Likewise, neuromuscular taping, an intervention that specifically targets body pain, was shown to be effective in pain relief among patients, in addition to the alleviation of fatigue and improvement of QOL [47]. These observations suggest that the development of multimodal interventions comprising multiple intervention strategies that were known to target a particular symptom could be more effective for symptom management.

This review has three limitations. First, only studies published in English were included in this review. Therefore, studies that could contribute further useful data to this review, yet not published in English were neglected, thereby limiting the comprehensiveness of this review. Second, the majority of the included studies have a weak overall rating in the appraisal on their methodological quality. With each of the categories in the appraisal having a potential in causing bias, the review findings 
have to be interpreted with caution. Third, several included studies utilized a small sample of about 30 participants. These studies would therefore be prone to small sample bias, a source for false negative results that lead to difficulties in drawing firm conclusions regarding the effects of the reported interventions [55].

\section{Implications}

Our review has suggested that various types of interventions, pharmacological and non-pharmacological alike, could be of value for implementation to help breast cancer patients undergoing adjuvant endocrine therapy to manage symptoms and improve QOL. Moreover, they suggest the benefit of using a multimodal approach in intervention design for addressing multiple adjuvant endocrine therapy-induced symptoms. Our findings thus provide an informative basis for further research into the effective approaches that would ameliorate symptoms induced by adjuvant endocrine therapy. This may improve adherence in breast cancer survivors undergoing adjuvant endocrine therapy, enabling it to be more effective in preventing cancer recurrence.

\section{Conclusions}

Our review shows that both pharmacological and non-pharmacological interventions can be effective for symptom management among breast cancer survivors undergoing adjuvant endocrine therapy, potentially enhancing their QOL. Nevertheless, firm conclusions cannot be drawn from the review over the types of interventions that are most optimal for supportive care of breast cancer survivors and highlights the need for a multimodal approach. This is due to the discrepancies in the findings between the included studies over the significance of the between-group differences in the extent of improvement of symptoms and QOL. Despite this, our review suggests that a longer duration in physical activity interventions could be more effective for symptom management. Further studies on whether the duration of physical activity interventions, and potentially that of other reported non-pharmacological interventions such as acupuncture, would be associated with intervention effectiveness on improving symptoms and QOL are therefore recommended. Moreover, these studies should involve the use of larger sample sizes for the generation of more reliable results. Overall, these studies would help contribute to the evidence of the utility of these interventions for symptom management among survivors undergoing these therapies.

Author Contributions: C.W.H.C. set the aim and focus of the manuscript. D.T., S.K., and B.M.H.L. conducted the literature search, data extraction, and critical appraisal of the included studies. D.T., S.K., and B.M.H.L. drafted the manuscript. C.W.H.C., K.M.C., and D.N.S.C. critically reviewed and revised the manuscript and provided advice on the presentation of the review. All authors have read and agreed to the published version of the manuscript.

Funding: The Chinese University of Hong Kong funded the open access publication cost of the manuscript.

Conflicts of Interest: The authors declare that no conflicts of interest exist.

\section{References}

1. Hershman, D.L.; Shao, T.; Kushi, L.H.; Buono, D.; Tsai, W.Y.; Fehrenbacher, L.; Kwan, M.; Gomez, S.L.; Neugut, A.I. Early discontinuation and non-adherence to adjuvant hormonal therapy are associated with increased mortality in women with breast cancer. Breast Cancer Res. Treat. 2011, 126, 529-537. [CrossRef] [PubMed]

2. Harbeck, N.; Gnant, M. Breast Cancer. Lancet 2017, 389, 1134-1150. [CrossRef]

3. Chan, C.W.H.; Law, B.M.H.; So, W.K.W.; Chow, K.M.; Waye, M.M.Y. Novel Strategies on Personalized Medicine for Breast Cancer Treatment: An Update. Int. J. Mol. Sci. 2017, 18, 2423. [CrossRef] [PubMed]

4. Haque, R.; Ahmed, S.A.; Fisher, A.; Avila, C.C.; Shi, J.; Guo, A.; Craig Cheetham, T.; Schottinger, J.E. Effectiveness of aromatase inhibitors and tamoxifen in reducing subsequent breast cancer. Cancer Med. 2012, 1, 318-327. [CrossRef] [PubMed] 
5. Early Breast Cancer Trialists' Collaborative Group (EBCTCG). Effects of chemotherapy and hormonal therapy for early breast cancer on recurrence and 15-year survival: An overview of the randomised trials. Lancet 2005, 365, 1687-1717. [CrossRef]

6. Gotay, C.; Dunn, J. Adherence to long-term adjuvant hormonal therapy for breast cancer. Expert Rev. Pharm. Outcomes Res. 2011, 11, 709-715. [CrossRef]

7. Van Herk-Sukel, M.P.; van de Poll-Franse, L.V.; Voogd, A.C.; Nieuwenhuijzen, G.A.; Coebergh, J.W.; Herings, R.M. Half of breast cancer patients discontinue tamoxifen and any endocrine treatment before the end of the recommended treatment period of 5 years: A population-based analysis. Breast Cancer Res. Treat. 2010, 122, 843-851. [CrossRef]

8. Miaskowski, C.; Shockney, L.; Chlebowski, R.T. Adherence to oral endocrine therapy for breast cancer: A nursing perspective. Clin. J. Oncol. Nurs. 2008, 12, 213-221. [CrossRef]

9. Garreau, J.R.; Delamelena, T.; Walts, D.; Karamlou, K.; Johnson, N. Side effects of aromatase inhibitors versus tamoxifen: The patients' perspective. Am. J. Surg. 2006, 192, 496-498. [CrossRef]

10. Henry, N.L.; Giles, J.T.; Ang, D.; Mohan, M.; Dadabhoy, D.; Robarge, J.; Hayden, J.; Lemler, S.; Shahverdi, K.; Powers, P.; et al. Prospective characterization of musculoskeletal symptoms in early stage breast cancer patients treated with aromatase inhibitors. Breast Cancer Res. Treat. 2008, 111, 365-372. [CrossRef]

11. Fallowfield, L.J.; Kilburn, L.S.; Langridge, C.; Snowdon, C.F.; Bliss, J.M.; Coombes, R.C.; IES Trial Steering Committee. Long-term assessment of quality of life in the Intergroup Exemestane Study: 5 years post-randomisation. Br. J. Cancer 2012, 106, 1062-1067. [CrossRef]

12. Schover, L.R.; Baum, G.P.; Fuson, L.A.; Brewster, A.; Melhem-Bertrandt, A. Sexual problems during the first 2 years of adjuvant treatment with aromatase inhibitors. J. Sex. Med. 2014, 11, 3102-3111. [CrossRef] [PubMed]

13. Awan, A.; Esfahani, K. Endocrine therapy for breast cancer in the primary care setting. Curr. Oncol. 2018, 25, 285-291. [CrossRef] [PubMed]

14. Khan, Q.J.; O'Dea, A.P.; Sharma, P. Musculoskeletal adverse events associated with adjuvant aromatase inhibitors. J. Oncol. 2010, 2010, 654348. [CrossRef] [PubMed]

15. Aiello Bowles, E.J.; Boudreau, D.M.; Chubak, J.; Yu, O.; Fujii, M.; Chestnut, J.; Buist, D.S. Patient-reported discontinuation of endocrine therapy and related adverse effects among women with early-stage breast cancer. J. Oncol. Pract. 2012, 8, e149-e157. [CrossRef]

16. Makubate, B.; Donnan, P.T.; Dewar, J.A.; Thompson, A.M.; McCowan, C. Cohort study of adherence to adjuvant endocrine therapy, breast cancer recurrence and mortality. Br. J. Cancer 2013, 108, 1515-1524. [CrossRef]

17. McCowan, C.; Wang, S.; Thompson, A.M.; Makubate, B.; Petrie, D.J. The value of high adherence to tamoxifen in women with breast cancer: A community-based cohort study. Br. J. Cancer 2013, 109, 1172-1180. [CrossRef]

18. Simpson, B. Hot flash pharmacotherapy in breast cancer survivors: A literature review. Can. Pharm. J. 2004, 137, 36-45.

19. Coleman, R.E.; Bolten, W.W.; Lansdown, M.; Dale, S.; Jackisch, C.; Merkel, D.; Maass, N.; Hadji, P. Aromatase inhibitor-induced arthralgia: Clinical experience and treatment recommendations. Cancer Treat. Rev. 2008, 34, 275-282. [CrossRef]

20. Bae, K.; Yoo, H.S.; Lamoury, G.; Boyle, F.; Rosenthal, D.S.; Oh, B. Acupuncture for Aromatase Inhibitor-Induced Arthralgia: A Systematic Review. Integr. Cancer Ther. 2015, 14, 496-502. [CrossRef]

21. Chen, L.; Lin, C.C.; Huang, T.W.; Kuan, Y.C.; Huang, Y.H.; Chen, H.C.; Kao, C.Y.; Su, C.M.; Tam, K.W. Effect of acupuncture on aromatase inhibitor-induced arthralgia in patients with breast cancer: A meta-analysis of randomized controlled trials. Breast 2017, 33, 132-138. [CrossRef]

22. Rada, G.; Capurro, D.; Pantoja, T.; Corbalán, J.; Moreno, G.; Letelier, L.M.; Vera, C. Non-hormonal interventions for hot flushes in women with a history of breast cancer. Cochrane Database Syst. Rev. 2010, CD004923. [CrossRef] [PubMed]

23. Chan, C.W.H.; Au Yeung, E.; Law, B.M.H. Effectiveness of Physical Activity Interventions on Pregnancy-Related Outcomes among Pregnant Women: A Systematic Review. Int. J. Environ. Res. Public Health 2019, 16, 1840. [CrossRef]

24. Quality Assessment Tool for Quantitative Studies Dictionary. Available online: https://merst.ca/wp-content/ uploads/2018/02/qualilty-assessment-dictionary_2017.pdf (accessed on 14 March 2020). 
25. De Sousa Vieira, A.F.; Mauad, V.A.Q.; Cubero, D.; Santi, P.X.; del Giglio, A. Use of Guarana for Hot Flashes after Breast Cancer: Randomized, Double-Blind, Placebo-Controlled Phase II Trial. J. Nat. Remedies 2019, 19, 1-11. [CrossRef]

26. Heudel, P.E.; Van Praagh-Doreau, I.; Duvert, B.; Cauvin, I.; Hardy-Bessard, A.C.; Jacquin, J.P.; Stefani, L.; Vincent, L.; Dramais, D.; Guastalla, J.P.; et al. Does a homeopathic medicine reduce hot flushes induced by adjuvant endocrine therapy in localized breast cancer patients? A multicenter randomized placebo-controlled phase III trial. Support. Care Cancer 2019, 27, 1879-1889. [CrossRef] [PubMed]

27. Kimmick, G.G.; Lovato, J.; McQuellon, R.; Robinson, E.; Muss, H.B. Randomized, double-blind, placebo-controlled, crossover study of sertraline (Zoloft) for the treatment of hot flashes in women with early stage breast cancer taking tamoxifen. Breast J. 2006, 12, 114-122. [CrossRef] [PubMed]

28. Pandya, K.J.; Raubertas, R.F.; Flynn, P.J.; Hynes, H.E.; Rosenbluth, R.J.; Kirshner, J.J.; Pierce, H.I.; Dragalin, V.; Morrow, G.R. Oral clonidine in postmenopausal patients with breast cancer experiencing tamoxifen-induced hot flashes: A University of Rochester Cancer Center Community Clinical Oncology Program study. Ann. Intern. Med. 2000, 132, 788-793. [CrossRef]

29. Goldberg, R.M.; Loprinzi, C.L.; O’Fallon, J.R.; Veeder, M.H.; Miser, A.W.; Mailliard, J.A.; Michalak, J.C.; Dose, A.M.; Rowland, K.M., Jr.; Burnham, N.L. Transdermal clonidine for ameliorating tamoxifen-induced hot flashes. J. Clin. Oncol. 1994, 12, 155-158. [CrossRef]

30. Mann, E.; Smith, M.J.; Hellier, J.; Balabanovic, J.A.; Hamed, H.; Grunfeld, E.A.; Hunter, M.S. Cognitive behavioural treatment for women who have menopausal symptoms after breast cancer treatment (MENOS 1): A randomised controlled trial. Lancet Oncol. 2012, 13, 309-318. [CrossRef]

31. Keshavarzi, Z.; Janghorban, R.; Alipour, S.; Tahmasebi, S.; Jokar, A. The effect of vitamin D and E vaginal suppositories on tamoxifen-induced vaginal atrophy in women with breast cancer. Support. Care Cancer 2019, 27, 1325-1334. [CrossRef]

32. Paulo, T.R.S.; Rossi, F.E.; Viezel, J.; Tosello, G.T.; Seidinger, S.C.; Simões, R.R.; de Freitas, R., Jr.; Freitas, I.F., Jr. The impact of an exercise program on quality of life in older breast cancer survivors undergoing aromatase inhibitor therapy: A randomized controlled trial. Health Qual. Life Outcomes 2019, 17, 17. [CrossRef] [PubMed]

33. Advani, P.; Brewster, A.M.; Baum, G.P.; Schover, L.R. A pilot randomized trial to prevent sexual dysfunction in postmenopausal breast cancer survivors starting adjuvant aromatase inhibitor therapy. J. Cancer Surviv. 2017, 11, 477-485. [CrossRef]

34. Henry, N.L.; Unger, J.M.; Schott, A.F.; Fehrenbacher, L.; Flynn, P.J.; Prow, D.M.; Sharer, C.W.; Burton, G.V.; Kuzma, C.S.; Moseley, A.; et al. Randomized, Multicenter, Placebo-Controlled Clinical Trial of Duloxetine Versus Placebo for Aromatase Inhibitor-Associated Arthralgias in Early-Stage Breast Cancer: SWOG S1202. J. Clin. Oncol. 2018, 36, 326-332. [CrossRef]

35. Nyrop, K.A.; Callahan, L.F.; Cleveland, R.J.; Arbeeva, L.L.; Hackney, B.S.; Muss, H.B. Randomized Controlled Trial of a Home-Based Walking Program to Reduce Moderate to Severe Aromatase Inhibitor-Associated Arthralgia in Breast Cancer Survivors. Oncologist 2017, 22, 1238-1249. [CrossRef] [PubMed]

36. Rogers, L.Q.; Hopkins-Price, P.; Vicari, S.; Markwell, S.; Pamenter, R.; Courneya, K.S.; Hoelzer, K.; Naritoku, C.; Edson, B.; Jones, L.; et al. Physical activity and health outcomes three months after completing a physical activity behavior change intervention: Persistent and delayed effects. Cancer Epidemiol Biomark. Prev. 2009, 18, 1410-1418. [CrossRef] [PubMed]

37. Rogers, L.Q.; Hopkins-Price, P.; Vicari, S.; Pamenter, R.; Courneya, K.S.; Markwell, S.; Verhulst, S.; Hoelzer, K.; Naritoku, C.; Jones, L.; et al. A randomized trial to increase physical activity in breast cancer survivors. Med. Sci. Sports Exerc. 2009, 41, 935-946. [CrossRef]

38. Irwin, M.L.; Cartmel, B.; Gross, C.P.; Ercolano, E.; Li, F.; Yao, X.; Fiellin, M.; Capozza, S.; Rothbard, M.; Zhou, Y.; et al. Randomized exercise trial of aromatase inhibitor-induced arthralgia in breast cancer survivors. J. Clin. Oncol. 2015, 33, 1104-1111. [CrossRef]

39. Hershman, D.L.; Unger, J.M.; Crew, K.D.; Awad, D.; Dakhil, S.R.; Gralow, J.; Greenlee, H.; Lew, D.L.; Minasian, L.M.; Till, C.; et al. Randomized Multicenter Placebo-Controlled Trial of Omega-3 Fatty Acids for the Control of Aromatase Inhibitor-Induced Musculoskeletal Pain: SWOG S0927. J. Clin. Oncol. 2015, 33, 1910-1917. [CrossRef] 
40. Hershman, D.L.; Unger, J.M.; Greenlee, H.; Capodice, J.L.; Lew, D.L.; Darke, A.K.; Kengla, A.T.; Melnik, M.K.; Jorgensen, C.W.; Kreisle, W.H.; et al. Effect of Acupuncture vs Sham Acupuncture or Waitlist Control on Joint Pain Related to Aromatase Inhibitors Among Women With Early-Stage Breast Cancer: A Randomized Clinical Trial. JAMA 2018, 320, 167-176. [CrossRef]

41. Oh, B.; Kimble, B.; Costa, D.S.; Davis, E.; McLean, A.; Orme, K.; Beith, J. Acupuncture for treatment of arthralgia secondary to aromatase inhibitor therapy in women with early breast cancer: Pilot study. Acupunct. Med. 2013, 31, 264-271. [CrossRef]

42. Crew, K.D.; Capodice, J.L.; Greenlee, H.; Apollo, A.; Jacobson, J.S.; Raptis, G.; Blozie, K.; Sierra, A.; Hershman, D.L. Pilot study of acupuncture for the treatment of joint symptoms related to adjuvant aromatase inhibitor therapy in postmenopausal breast cancer patients. J. Cancer Surviv. 2007, 1, 283-291. [CrossRef] [PubMed]

43. Baker, M.K.; Peddle-McIntyre, C.J.; Galvão, D.A.; Hunt, C.; Spry, N.; Newton, R.U. Whole Body Vibration Exposure on Markers of Bone Turnover, Body Composition, and Physical Functioning in Breast Cancer Patients Receiving Aromatase Inhibitor Therapy: A Randomized Controlled Trial. Integr. Cancer Ther. 2018, 17, 968-978. [CrossRef] [PubMed]

44. Zhao, H.; Zhang, Q.; Zhao, L.; Huang, X.; Wang, J.; Kang, X. Spore Powder of Ganoderma lucidum Improves Cancer-Related Fatigue in Breast Cancer Patients Undergoing Endocrine Therapy: A Pilot Clinical Trial. Evid. Based Complement Alternat. Med. 2012, 2012, 809614. [CrossRef] [PubMed]

45. Peppone, L.J.; Janelsins, M.C.; Kamen, C.; Mohile, S.G.; Sprod, L.K.; Gewandter, J.S.; Kirshner, J.J.; Gaur, R.; Ruzich, J.; Esparaz, B.T.; et al. The effect of YOCAS@ßyoga for musculoskeletal symptoms among breast cancer survivors on hormonal therapy. Breast Cancer Res. Treat. 2015, 150, 597-604. [CrossRef]

46. Mao, J.J.; Farrar, J.T.; Bruner, D.; Zee, J.; Bowman, M.; Seluzicki, C.; DeMichele, A.; Xie, S.X. Electroacupuncture for fatigue, sleep, and psychological distress in breast cancer patients with aromatase inhibitor-related arthralgia: A randomized trial. Cancer 2014, 120, 3744-3751. [CrossRef]

47. Conejo, I.; Pajares, B.; Alba, E.; Cuesta-Vargas, A.I. Effect of neuromuscular taping on musculoskeletal disorders secondary to the use of aromatase inhibitors in breast cancer survivors: A pragmatic randomised clinical trial. BMC Complement. Altern. Med. 2018, 18, 180. [CrossRef]

48. Rogers, L.Q.; Courneya, K.S.; Oster, R.A.; Anton, P.M.; Robbs, R.S.; Forero, A.; McAuley, E. Physical Activity and Sleep Quality in Breast Cancer Survivors: A Randomized Trial. Med. Sci. Sports Exerc. 2017, 49, 2009-2015. [CrossRef]

49. Cheng, K.K.F.; Thompson, D.R.; Ling, W.M.; Chan, C.W.H. Measuring symptom prevalence, severity and distress of cancer survivors. Clin. Eff. Nurs. 2006, 9, 154-160. [CrossRef]

50. Chen, D.; Shiou, W.; Wang, K.; Huang, S.; Shie, Y.; Tsai, C.; Shie, J.; Chen, K. Chemotaxonomy of Triterpenoid Pattern of HPLC of Ganoderma lucidum and Ganoderma tsugae. J. Chin. Chem Soc. 1999, 46, 47-51. [CrossRef]

51. Zhu, M.; Chang, Q.; Wong, L.K.; Chong, F.S.; Li, R.C. Triterpene antioxidants from ganoderma lucidum. Phytother Res. 1999, 13, 529-531. [CrossRef]

52. Geng, P.; Siu, K.C.; Wang, Z.; Wu, J.Y. Antifatigue Functions and Mechanisms of Edible and Medicinal Mushrooms. Biomed. Res. Int. 2017, 2017, 9648496. [CrossRef] [PubMed]

53. Becker, W.C.; Dorflinger, L.; Edmond, S.N.; Islam, L.; Heapy, A.A.; Fraenkel, L. Barriers and facilitators to use of non-pharmacological treatments in chronic pain. BMC Fam. Pract. 2017, 18, 41. [CrossRef] [PubMed]

54. Nakano, J.; Hashizume, K.; Fukushima, T.; Ueno, K.; Matsuura, E.; Ikio, Y.; Ishii, S.; Morishita, S.; Tanaka, K.; Kusuba, Y. Effects of Aerobic and Resistance Exercises on Physical Symptoms in Cancer Patients: A Meta-analysis. Integr. Cancer Ther. 2018, 17, 1048-1058. [CrossRef]

55. Biau, D.J.; Kernéis, S.; Porcher, R. Statistics in brief: The importance of sample size in the planning and interpretation of medical research. Clin. Orthop. Relat. Res. 2008, 466, 2282-2288. [CrossRef] [PubMed]

(C) 2020 by the authors. Licensee MDPI, Basel, Switzerland. This article is an open access article distributed under the terms and conditions of the Creative Commons Attribution (CC BY) license (http://creativecommons.org/licenses/by/4.0/). 